\title{
Non-Isothermal Spreading of Liquid Drops on Horizontal Plates
}

\author{
P. Ehrhard, S.H. Davis \\ Institut für Reaktorbauelemente
}

\section{Kernforschungszentrum Karlsruhe}



KERNFORSCHUNGSZENTRUM KARLSRUHE

Institut für Reaktorbauelemente

KfK 4720

Non-Isothermal Spreading of Liquid Drops on Horizontal Plates

P. Ehrhard, S.H. Davis*)

*) Department of Engineering Sciences and Applied Mathematics McCormick School of Engineering and Applied Science

Northwestern University, Evanston, IL 60208, USA

Kernforschungszentrum Karlsruhe $\mathrm{GmbH}$, Karlsruhe 



\begin{abstract}
A viscous-liquid drop spreads on a smooth horizontal surface, which is uniformly heated or cooled. Lubrication theory is used to study thin drops subject to capillary, thermocapillary and gravity forces, and a variety of contact-angle-versus-speed conditions. It is found for isothermal drops that gravity is very important at large times and determines the power law for unlimited spreading. Predictions compare well with the experimental data on isothermal spreading for both two-dimensional and axisymmetric configurations. It is found that heating (cooling) retards (augments) the spreading process. When the advancing contact angle is zero, heating will cause the drop to spread only finitely far. For positive advancing contact angles, sufficient cooling will cause unlimited spreading. Thus, the heat transfer serves as a sensitive control on the spreading.
\end{abstract}




\section{Ausbreitung eines Flüssigkeitstropfens auf einer horizontalen, temperierten Platte}

\section{Zusammenfassung:}

Bringt man eine viskose Flüssigkeit auf eine glatte horizontale Platte, so breitet sich der geformte Tropfen unter bestimmten Bedingungen aus. Hierbei wird zusätzlich der Einfluss einer gleichmäßigen Beheizung oder Kühlung der Platte berücksichtigt. Somit sind am Tropfen die Kapillaritäts-, die Thermokapillaritäts- und die Schwerkraft erfaßt; die Bedingung an der bewegten Kontaktlinie wird im Modell durch ein universelles Potenzgesetzt für den Kontaktwinkel als Funktion der Geschwindigkeit der Kontaktlinie eingebracht. Wir beschränken uns auf "dünne" Tropfen, d.h. der anfängliche Kontaktwinkel wird als klein angenommen (Schmierschicht-Approximation).

Die Ergebnisse der Analytik zeigen, daß im isothermen Fall für große Zeiten die Schwerkraft den Prozess kontrolliert und somit bei unbegrenzter Ausbreitung das Zeitgesetz bestimmt. Für beide Geometrien, eben oder achsensymmetrisch, befinden sich unsere Ergebnisse in guter Übereinstimmung mit experimentellen Resultaten anderer Authoren.

Wird die Platte beheizt (gekühlt), so läuft der Ausbreitungsvorgang langsamer (schneller) ab als im vergleichbaren isothermen Fall. Ein Vergleich dieser Modellaussagen mit Experimenten ist derzeit nicht möglich - thermisch kontrollierte Tropfenausbreitungen sind offenbar nicht durchgeführt. Für einen statischen Kontaktwinkel von $\boldsymbol{\Theta}_{\mathrm{A}}=0$ führt die Beheizung der Platte dazu, daß der Tropfen sich lediglich bis zu einer endlichen Größe ausbreitet. Umgekehrt kann bei einem positiven statischen Kontaktwinkel $\Theta_{A}>0$ eine ausreichende Kühlung der Platte zu unbegrenzter Ausbreitung führen. Somit kann die Temperierung der Platte als wirksames Instrument zur Kontrolle des Ausbreitungsvorgangs aufgefaßt werden. 


\section{Contents:}

1. Introduction 1

2. Formulation 5

3. Scaling and the lubrication approximation 11

4. Derivation of the evolution equation 15

5. The $C \rightarrow 0$ problem 19

6. Results: Isothermal spreading 21

7. Results: Nonisothermal spreading 27

8. Discussion and conclusion 33

$\begin{array}{ll}\text { References } & 36\end{array}$

$\begin{array}{ll}\text { Table } & 37\end{array}$

$\begin{array}{ll}\text { Figure Captions } & 38\end{array}$

$\begin{array}{ll}\text { Figures } & 40\end{array}$ 


\section{Introduction}

The spreading of a liquid on a smooth solid is a fundamental problem in fluid mechanics. It exemplifies the general problem of moving contact lines which enters a host of applications such as coating technology, mold filling and the performance in space of fuel tanks. It involves the modeling of the local mechanics near the contact lines whenever a continuum theory of the motion is employed. A spreading drop involves bulk, surface and line forces intrinsically coupled through a free-boundary problem. The configuration is shown in Figure 1.

Body forces affect the drop in a classical sense; gravity will greatly promote the spreading if the hydrostatic head is appreciable.

Surface forces enter the description through the 1iquid/gas interface on which surface tension acts. Further, if the contact line moves, and the no slip condition is applied on the solid/liquid interface, then, as shown by Dussan V. and Davis (1974), there is a force singularity at the contact line. The implication then is that on a continuum level there is effective slip at the liquid/solid interface. This is also a surface effect.

At the contact line there is a contact-angle condition. In the twodimensional case, the interface slope $h_{x}$ at the contact line at $x=a$ must satisfy the compatibility condition $h_{x}(a, t)=-\tan \theta$, where $\theta$ is the contact angle and the interface is located at $z=h(x, t)$, as shown in Figure 1 . One must specify a constitutive law for $\theta$, since it is here that the chemistry of the surfaces has an effect. If the interface moves with speed $U$, then one may specify $\theta=F(U)$; this determines the mobility of the contact line. Dussan $V$. (1979) discusses such models and the data that underlie them. The contactangle condition gives a line effect in the model description. 
There are a number of approaches to the modeling of the dynamics of spreading drops. These can be roughly categorized as (i) excision, (ii) microscopic and (iii) uniform analyses.

(i) Tanner (1979) considered viscous and capillary forces only and used lubrication theory to describe the bulk drop, away from the contact line. Analysis of the excised contact-line region is replaced by a priori statements about the "outer" balances and about the drop shape. For unlimited spreading, he found power laws for the drop radius as a function of time $t$ for large times. He found $a-t^{1 / 7}$ in two dimensions and $a-t^{1 / 10}$ in the axisymmetric case. Starov (1983) further extracted the multiplicative prefactor in these forms. Lopez, Miller and Ruckenstein (1976) took a similar approach, but ignored capillarity, and instead included gravity or long-range molecular forces near $\mathrm{x}=\mathrm{a}$. They sought similarity solutions and found for the gravity-dominated drop that $a \sim t^{1 / 5}$ in two dimensions and $a \sim t^{1 / 8}$ in the axisymmetric case. The authors pointed out that these similarity solutions gave infinite shear stresses at the contact lines; thus, the solutions break down at the points of interest. The case in which long-range forces dominate leads to thin films with no discernible leading edges.

(ii) Another approach to spreading is that of de Gennes (1985) who wished to examine the small-scale physics of contact lines. He included in his model long-range van der Waals repulsions and so obtained a drop that possesses no contact line nearby, but instead found a thick drop that smoothly blends into a "foot", an extended thin film from the main drop sometimes far forward along the plate. On the one hand there is no longer a contact line nearby to consider, and on the other hand he did not consider the actual contact line at the "foot's" edge. This approach may lead to useful information on the functional form of $F$, but its direct pursuit requires one 
to examine "feet" whose thicknesses are in the $10 \AA$ range; these are essentially invisible in a continuum theory.

(iii) There is a uniform approach to the continuum theory in which one considers the whole drop including the contact line, inserts local slip nearby and poses $\theta=F(U)$. Greenspan (1978) posed such a model including both capillary and viscous forces and with F linear. He used lubrication theory for flat drops and obtained an evolutionary system giving the drop history.

The present paper aims to analyze the spreading of a viscous drop on a smooth, horizontal plate. The approach generalizes that of Greenspan (1978) in three ways. (i) The angle-versus-speed characteristic is generalized to $\theta=\theta_{\mathrm{A}}+\kappa^{-1} \mathrm{U}^{1 / \mathrm{m}}$, where $\theta_{\mathrm{A}}$ is the (static) advancing contact angle and $\kappa$ is an empirical coefficient. We term the factor $m$ the mobility exponent. This form with $m=1$ was used by Greenspan, while the case $m=3$ is suggested by Schwartz and Tejeda (1972) using data on contact-line dynamics. (ii) Gravity, acting vertically downward, is included. (iii) The plate is uniformly heated or cooled, compared to the surrounding gas. If the plate is hotter than the surrounding air, then the non-isothermal liquid/gas interface will have the contact line hotter than the drop summit. Thermocapillarity will drive a flow in the viscous drop that will either augment or hinder the spreading. The heat transfer is conduction dominated in this lubrication limit.

The analysis obtains generalized evolution equations for the spreading in both two-dimensional and axisymmetric configurations. The equations are then examined in the limit of small capillary number in which spreading rates, interface shapes and velocity fields can be determined over the whole drop. A number of interesting results are obtained.

In the isothermal case solutions that include viscous forces, gravity and surface tension show how thin drops with negligible gravity effects can spread 
to such large size that gravity becomes important, as observed by Cazabat and Cohen Stuart (1986). When the static advancing angle $\theta_{A}=0$, the drop spreads to infinity. The power laws obtained here agree very well with those of previous theory and experiment, validating both the lubrication theory and the choices of the mobility exponent $m=3$.

In the nonisothermal case thermocapillary-driven flows alter the spreading process. For example, a drop with advancing contact angle $\theta_{A}=0$ will spread forever under isothermal conditions. The same drop, if $\theta_{A}=0$ still, will spread only finitely far if the plate is heated. Conversely, if $\theta_{A}>0$, the isothermal drop will spread and then come to rest at a finite size. However, if the plate is cooled sufficiently, and $\theta_{A}>0$ still, then it will spread forever. The heating or cooling can thus be used to control the spreading process. 


\section{Formulation}

Consider a drop of liquid on a smooth, horizontal rigid plane located at a position $z=0$ and kept at a constant temperature $T=T_{W}$. The drop is composed of a non-volatile Newtonian liquid with constant material properties and surrounded by a passive gas, whose viscosity and thermal conductivity are taken to be very small compared to those of the liquid; the far-field gas temperature is $\mathrm{T}_{\infty}$. The drop, shown in Figure 1, is either two-dimensional in Cartesian coordinates $(x, z)$ or axisymmetric in cylindrical coordinates $(r, z)$. We shall examine both cases and denote equation numbers by indices $p$ (plane) and a (axisymmetric), respectively. The shape of the interface between the spreading liquid and the ambient gas is denoted by $z=h$, and the position of the contact-line is given by either $x=a$ or $r=a$.

The velocity and thermal fields in the liquid are governed by the NavierStokes, the continuity, and the energy equations:

$$
\begin{aligned}
& \rho\left\{\frac{\partial \mathbf{v}}{\partial t}+\mathbf{v} \cdot \nabla \mathbf{v}\right\}=-\nabla p+\mu \nabla^{2} \mathbf{v}-\rho g \mathbf{k} \\
& \nabla \cdot \mathbf{v}=0, \\
& \rho c_{p}\left\{\frac{\partial T}{\partial t}+\mathbf{v} \cdot \nabla T\right\}=\lambda \nabla^{2} \mathrm{~T} .
\end{aligned}
$$

where $\mathbf{k}=(0,1), \mathbf{v}=(\mathrm{u}, \mathrm{w})$ is the velocity vector, $\mathrm{p}$ is the pressure and $\mathrm{T}$ is the temperature of the liquid. Here $\mathrm{g}$ is the magnitude of the gravitational acceleration, $\rho$ is the density, $\mu$ is the viscosity, $c_{p}$ is the specific heat, and $\lambda$ the heat conductivity of the liquid.

The equations (2.1)-(2.3) are subject to the following boundary conditions at the liquid/solid interface:

$$
z=0: \quad u=\beta^{\prime} \frac{\partial u}{\partial z}, \quad w=0, \quad T=T_{W} .
$$


The rigid plane is considered to be an impenetrable, perfectly-conducting material. Following Dussan V. and Davis (1974), the no-slip condition at the rigid boundary is relaxed to avoid the appearance of a shear-stress singularity at the moving contact line. The slip coefficient $\beta^{\prime}$ in equation $(2.4)$ is taken to be a small constant.

At the liquid/gas interface there are the following conditions:

$$
\begin{aligned}
z=h: \quad w-\frac{\partial h}{\partial t}=\frac{\partial h}{\partial x} u, \\
w-\frac{\partial h}{\partial t}=\frac{\partial h}{\partial r} u, \\
n \cdot T \cdot \mathbf{n}=2 H \sigma, \\
t \cdot T \cdot n=t \cdot \nabla \sigma, \\
-\lambda \frac{\partial T}{\partial n}=\frac{\lambda}{\delta}\left(T-T_{\infty}\right) .
\end{aligned}
$$

Here $\mathbf{T}$ denotes the stress tensor of the liquid, $\mathbf{n}$ and $\boldsymbol{t}$ are the unit normal and tangential vectors with $\mathbf{n}$ pointing out of the liquid (see Figure 1). Equation (2.5) is the kinematic condition while equations (2.6) and (2.7) give the dynamic conditions, balancing normally and tangentially, the stress components across the liquid/gas interface. The mean curvature $\mathrm{H}$ in equation (2.6) is given by

$$
2 \mathrm{H}=\nabla \cdot\left\{\left[1+|\nabla \mathrm{h}|^{2}\right]^{-1 / 2} \nabla \mathrm{h}\right\} .
$$

Here the surface tension $\sigma$ depends linearly on temperature

$$
\sigma(\mathrm{T})=\sigma_{\mathrm{W}}-\alpha\left(\mathrm{T}-\mathrm{T}_{\mathrm{W}}\right)
$$

$\alpha>0$, so that equation (2.7) incorporates the effects of thermocapillarity. The surface tension in equation (2.10) is denoted by $\sigma$ and $\sigma_{W}$ is the surface tension at a temperature $\mathrm{T}_{\mathrm{W}}$. 
The thermal boundary condition at the 1iquid/gas interface is chosen to be of third type, i.e., we use a mixed condition on the heat flux and the local temperature, involving the parameter group $\lambda_{\infty} / \lambda \delta$, which contains all limiting cases between an adiabatic and a perfect conducting boundary. Here $\lambda_{\infty}$ denotes the thermal conductivity of the ambient gas, while $\delta$ is the thickness of the thermal boundary layer established within the gas. Such a model may break down near the contact line when $h$ is smaller than $\delta$ but such a model is a reasonable one for an initial investigation.

We must specify initial conditions, symmetry or smoothness conditions, and volume constraints on the drop shape. These are as follows:

$$
\begin{aligned}
& h(x, 0)=h_{0}(x), \quad h_{0}\left(a_{0}\right)=0, a(0)=a_{0}, \\
& h(r, 0)=h_{0}(r), \quad h_{0}\left(a_{0}\right)=0, a(0)=a_{0}, \\
& \frac{\partial h_{0}}{\partial x}(0)=0, \frac{\partial^{3} h_{0}}{\partial x^{3}}(0)=0, \\
& \frac{\partial h_{0}}{\partial r}(0)=0, \lim _{r \rightarrow 0}\left\{r \frac{\partial^{3} h_{0}}{\partial x^{3}}(r)\right\}=0, \\
& \frac{\partial h_{0}}{\partial x}\left(a_{0}\right)=-\tan \theta_{0}, \\
& \frac{\partial h_{0}}{\partial r}\left(a_{0}\right)=-\tan \theta_{0},
\end{aligned}
$$

Thus, the drop is symmetric and smooth initially with an edge at $a_{0}$ and an initial contact angle of $\theta_{0}$. The volume of the drop is conserved during the spreading process and can be calculated from the initial drop shape. The volume per unit width in the two-dimensional case is given by 


$$
v_{0}=\int_{-a_{0}}^{a_{0}} h_{0}(x) d x
$$

while the volume in the axisymmetric case is given by

$$
v_{0}=2 \pi \int_{0}^{a_{0}} r h_{0}(r) d r
$$

For $t>0$ the drop retains its symmetries; the edge, symmetry, smoothness and volume conditions are thus given as follows:

$$
\begin{aligned}
& h[a(t), t]=0, \quad \text { (condition of contact) } \\
& \frac{\partial h}{\partial x}(0, t)=0, \frac{\partial^{3} h}{\partial x^{3}}(0, t)=0, \\
& \frac{\partial h}{\partial r}(0, t)=0, \lim _{r \rightarrow 0}\left\{r \frac{\partial^{3} h}{\partial r^{3}}(r, t)\right\}=0, \\
& \text { symmetry and } \\
& \text { smoothness conditions) } \\
& \left.\begin{array}{l}
\frac{\partial h}{\partial x}[a(t), t]=-\tan \theta(t), \\
\frac{\partial h}{\partial r}[a(t), t]=-\tan \theta(t),
\end{array}\right\} \\
& \text { (contact-angle condition) } \\
& \int_{-a(t)}^{a(t)} h(x, t) d x=v_{0} \\
& 2 \pi \int_{0}^{a(t)} r h(r, t) d r=v_{0}
\end{aligned}
$$

Based on various experimental results, Dussan V. (1981) discusses how the contact angle $\theta$ depends on the speed $a_{t}$ of the contact line. The following constitutive form is chosen here:

$$
a_{t}=\kappa\left(\theta-\theta_{A}\right)^{m} \quad, \quad m \geq 1
$$


where $\kappa>0$ is an empirical constant and $\theta_{A} \geq 0$ is the (static) advancing contact angle. The typical behavior of experimental data (from Dussan $V$. 1981) and the above model (2.19) for various $m$ are shown in Figure 2 . The form (2.19) for $m=3$ is suggested by the data of Schwartz and Tejeda (1972). 



\section{Scaling and the lubrication approximation}

The analysis of Greenspan (1978) uses lubrication theory to simplify the governing system for isothermal spreading. The present work generalizes that of Greenspan in three ways. (i) The effects of gravity are included. The contact-angle-versus-speed characteristic (2.19) allows for power-law behavior with exponent $m$; Greenspan used $m=1$. (iii) Non-isothermal spreading is allowed through the action of thermocapillarity.

We generalize the analysis of Greenspan (1978) by introducing the following set of dimensionless variables:

$$
\begin{aligned}
& x^{*}=\frac{x}{a_{0}}, r^{*}=\frac{r}{a_{0}}, z^{*}=\frac{z}{a_{0} \theta_{0}}, t^{*}=\frac{\kappa \theta_{0}^{m}}{a_{0}} t, \\
& u^{*}=\frac{u}{\kappa \theta_{0}^{m}}, \quad w^{*}=\frac{w}{\kappa \theta_{0}^{1+m}}, p^{*}=\frac{a_{0} \theta_{0}^{2-m}}{\mu \kappa} p, \\
& T^{*}=\frac{T-T_{\infty}}{T_{W}-T_{\infty}}, \theta=\frac{\theta}{\theta_{0}}, \quad v^{*}=\frac{v}{v_{0}} .
\end{aligned}
$$

Thus, the space variables $(x, z)$ or $(r, z)$ are scaled using the initial shape of the drop in horizontal and vertical directions, respectively. The time scale is constructed using the horizontal length scale $a_{0}$ together with an estimate of the initial speed of the contact line, $\kappa \theta_{0}^{\mathrm{m}}$, which is obtained by use of equation (2.19) for $\theta_{A}=0$. Conservation of mass determines the velocity scales. The pressure scale is obtained by balancing the pressure gradients and viscous terms in the horizontal component of the Navier-Stokes equations. The temperature scaling is chosen to allow the largest possible (unit order) temperature difference. 
If scalings, (3.1) are introduced into system (2.1)-(2.19), the leadingorder asymptotic equations valid as $\theta_{0} \rightarrow 0$ lead to the "lubrication approximation":

$$
\begin{aligned}
& -p_{x}+u_{z z}=0, \\
& -p_{I}+u_{z z}=0, \\
& -p_{z}-\frac{G}{C}=0, \\
& u_{x}+w_{z}=0, \\
& (r u)_{r}+r w_{z}=0, \\
& T_{z z}=0,
\end{aligned}
$$

where subscripts denote partial differentiation. For simplicity, all asterisks have been dropped. The boundary conditions are as follows:

$$
\begin{aligned}
& z=0: \quad u=\beta u_{z}, \quad w=0, \theta=1 ; \\
& z=h: \quad w-h_{t}=u h_{x}, \\
& w-h_{t}=u h_{r}, \\
& -C p=h_{x x}, \\
& -C p=h_{r r}+\frac{1}{r} h_{r}, \\
& \Delta C u_{z}=-\left(T_{x}+h_{x} T_{z}\right), \\
& \Delta C u_{z}=-\left(T_{r}+h_{r} T_{z}\right), \\
& T_{z}+B T=0 .
\end{aligned}
$$

Further, for $t=0$ :

$$
\begin{aligned}
& h(x, 0)=h_{0}(x), \quad h_{0}(1)=0, \quad a(0)=1, \\
& h(r, 0)=h_{0}(r), \quad h_{0}(1)=0, \quad a(0)=1, \\
& h_{0 x}(0)=0, \quad h_{0 \times x x}(0)=0,
\end{aligned}
$$




$$
\begin{aligned}
& h_{0 r}(0)=0, \lim _{r \rightarrow 0}\left[\mathrm{rh}_{0 \mathrm{rrr}}(r)\right]=0, \\
& h_{0 \mathrm{x}}(1)=-1, \\
& \mathrm{~h}_{0 \mathrm{r}}(1)=-1, \\
& 1=\int_{-1}^{1} h_{0}(\mathrm{x}) \mathrm{dx}, \\
& 1=2 \pi \int_{0}^{1} \mathrm{rh}_{0}(\mathrm{r}) \mathrm{dr},
\end{aligned}
$$

and, for $t>0$

$$
\begin{aligned}
& h(a, t)=0, \\
& h_{x}(0, t)=0, h_{x x x}(0, t)=0, \\
& h_{r}(0, t)=0, \lim _{r \rightarrow 0}\left[r h_{r r r}(r, t)\right]=0, \\
& h_{x}(a, t)=-\theta(t), \\
& h_{r}(a, t)=-\theta(t), \\
& I=\int_{-a(t)}^{a(t)} h(x, t) d x, \\
& 1=2 \pi \int_{0}^{a(t)} \operatorname{rh}(r, t) d r .
\end{aligned}
$$

The instantaneous contact angle $\theta(t)$ is expressed in terms of the dimensionless speed $a_{t}$ of the contact line, i.e.

$$
\theta(t)=\left[a_{t}(t)\right]^{1 / m}+\theta_{A}
$$

A number of dimensionless parameters arise. These are the capillary number $C$, the Bond number $G$, the thermocapillary number $\Delta C$, the Biot number $B$, as well as the slip coefficient $\beta$. Their definitions are as follows: 


$$
\begin{aligned}
& \mathrm{C}=\frac{\mu_{0} \kappa}{\sigma_{\mathrm{W}} \theta_{0}^{3-\mathrm{m}}}, \quad \mathrm{G}=\frac{\rho \mathrm{ga}_{0}^{2}}{\sigma_{\mathrm{W}}}, \quad \mathrm{B}=\frac{\lambda_{\infty} \mathrm{a}_{0} \theta_{0}}{\lambda \delta}, \\
& \Delta \mathrm{C}=\frac{\mu_{0} \kappa}{\alpha\left(\mathrm{T}_{\mathrm{W}}-\mathrm{T}_{\infty}\right) \theta_{0}^{1-\mathrm{m}}}, \quad \beta=\frac{\beta^{\prime}}{\mathrm{a}_{0} \theta_{0}} .
\end{aligned}
$$

The capillary number compares dissipative effects to mean surface tension. The Bond number relates gravity forces to mean surface tension. The thermocapillary number measures the fractional thermal change in the surface tension. Finally, the Biot number characterizes the quality of the heat transfer occurring at the 1iquid/gas interface. In particular $B \rightarrow 0$ and $B \rightarrow \infty$ give the adiabatic and the perfect-conducting limits, respectively.

The simplified Navier Stokes equations (3.2) and (3.3) show that the horizontal pressure gradient is balanced by the viscous shear stresses, while vertically there is a hydrostatic balance. From the energy equation (3.5) we see that heat is transported across the drop mainly by conduction. 


\section{Derivation of the evolution equation}

Within the lubrication approximation the Navier-Stokes equations (3.2) and (3.3) and the continuity equation (3.4), decouple from the heat transport. Therefore, one can solve for the thermal field, governed by equation (3.5) and subject to the thermal boundary conditions (3.6c) and (3.10) to obtain

$$
T=\frac{1+B(h-z)}{1+B h}
$$

The position of the liquid/gas interface, $h(x, t)$ or $h(r, t)$ is unknown a priori, and through $h$ the temperature will depend on both space coordinates as well as on time. The interface temperature can be found from equation (4.1) as

$$
T(h)=\frac{1}{1+B h} .
$$

Equation (4.2) shows for both the adiabatic and the perfectly-conducting limits that the temperature along the liquid/gas interface is constant. In fact,

$$
\begin{aligned}
& B \rightarrow 0: T(h)=1, \\
& B+\infty: T(h)=0 .
\end{aligned}
$$

The adiabatic limit results in the interface temperature $\mathrm{T}_{\mathrm{W}}$, while the perfectly-conducting limit gives $T_{\infty}$. In order to have variations in temperature and therefore surface-tension gradients along the interface, we must have the Biot number in the range $0<B<\infty$.

We use continuity equation (3.4) and integrate across the liquid layer, using the appropriate boundary conditions (3.6b) and (3.7) to obtain

$$
\begin{aligned}
& h_{t}+\frac{\partial}{\partial x} \int_{0}^{h} u d z=0 \\
& h_{t}+\frac{\partial}{\partial r} \int_{0}^{h} u d z=0
\end{aligned}
$$


The integration of the momentum equation (3.3) and application of the normalstress boundary condition (3.8) gives

$$
\begin{aligned}
& h_{x x}+C p(x, 0, t)-G h=0, \\
& h_{r r}+\frac{1}{r} h_{r}+C p(r, 0, t)-G h=0 .
\end{aligned}
$$

The horizontal momentum equation (3.2) is integrated twice with respect to $z$ and the boundary conditions (3.6a) and (3.9) are applied, to obtain

$$
\begin{aligned}
& C \int_{0}^{h} u d z=\left\{\frac{h^{3}}{3}+\beta h^{2}\right\} D_{3 x} h+\frac{C}{\Delta C} \frac{B}{(1+B h)^{2}}\left\{\frac{h^{2}}{2}+\beta h\right\} h_{x}, \\
& \text { C } \frac{1}{r} \int_{0}^{h}(r u) d z=\left\{\frac{h^{3}}{3}+\beta h^{2}\right\} D_{3 r} h+\frac{C}{\Delta C} \frac{B}{(1+B h)^{2}}\left\{\frac{h^{2}}{2}+\beta h\right\} h_{r},
\end{aligned}
$$

Here the operators $D_{3 x}$ and $D_{3 r}$ are defined as

$$
\begin{aligned}
& D_{3 x} h=\left\{h_{x x}-G h\right\}_{x} . \\
& D_{3 r} h=\left\{h_{r r}+\frac{1}{r} h_{r}-G h\right\}_{r} .
\end{aligned}
$$

Equations (4.4) and (4.6) then provide an evolution equation for the drop shape as follows:

$$
\begin{aligned}
& \mathrm{Ch}_{t}+\left\{\left[\frac{h^{3}}{3}+\beta h^{2}\right] D_{3 x} h+\frac{C}{\Delta C} \frac{B}{(1+B h)^{2}}\left[\frac{h^{2}}{2}+\beta h\right] h_{x}\right\}_{x}=0 . \\
& C h_{t}+\frac{1}{r}\left\{\left[\frac{h^{3}}{3}+\beta h^{2}\right] r D_{3 r} h+\frac{C}{\Delta C} \frac{B}{(1+B h)^{2}}\left[\frac{h^{2}}{2}+\beta h\right]_{r h}\right\}_{r}=0 .
\end{aligned}
$$

The evolution equation (4.8) allows us to bypass the free-boundary problem for $h$, though the edge position $a(t)$ is still unknown. The edge, symmetry and volume condition on the drop shape, equations (3.11)-(3.19), still apply.

In what follows, the Biot number will be considered to be much smaller than unity 


$$
B<1
$$

so that the effect of thermocapillarity, as shown in evolution equations (4.8), is incorporated only in the product $C B / \triangle C$, this serves as the effective Marangoni number $M$,

$$
M=\frac{C B}{\Delta C} \text {. }
$$

When $M>0$, the plate is heated with respect to the gas, while is $M<0$, it is cooled.

\section{The flow field}

After the solutions of the evolution equation (4.8) are known, one can calculate the velocity field:

$$
\begin{aligned}
& C u=\left[h(z+\beta)-\frac{z^{2}}{2}\right] D_{3 x} h+M h_{x}(z+\beta), \\
& C w=\left[\frac{z^{3}}{6}-\frac{z^{2}}{2} h-\beta h z\right] D_{4 x} h-\left[\frac{z^{2}}{2}+\beta z\right] h_{x} D_{3 x} h \\
& C u=\left[\frac{z^{2}}{2}+\beta z\right] h_{x x}, \\
& C w=\left[\frac{z^{3}}{6}-\frac{z^{2}}{2} h-\beta h z\right] D_{4 r} h-\left[\frac{z^{2}}{2}+\beta z\right] h_{r} D_{3 r} h \\
& \left.C(z+\beta)-\frac{z^{2}}{2}\right] D_{3 r} h+M h_{r}(z+\beta), \\
& C M\left[\frac{z^{2}}{2}+\beta z\right] \frac{1}{r}\left(r h_{r}\right)_{r}
\end{aligned}
$$

where the operators $\mathrm{D}_{4 \mathrm{x}}$ and $\mathrm{D}_{4 \mathrm{r}}$ are defined by

$$
\begin{aligned}
& D_{4 x} h=\frac{\partial}{\partial x}\left(D_{3 x} h\right), \\
& D_{4 r} h=\frac{1}{r} \frac{\partial}{\partial r}\left[r\left(D_{3 r} h\right)\right] .
\end{aligned}
$$





\section{The $\mathrm{C} \rightarrow 0$ problem}

In this paper only the quasi-steady limit, $\mathrm{C} \rightarrow 0$, will be analyzed. As discussed by Rosenblat and Davis (1985), the dropping of the unsteady term in equation (4.8) leads to an "outer" solution in time. The initial condition on $h$ must be dropped while that on $a(t)$ is enforced. The unsteady term $a_{t}$ in the contact-line conditions (3.17) and (3.19), is, of course, retained since this allows the drop to evolve. Details of the analysis are shown for the twodimensional drop. The details are similar for the axisymmetric drop and only results are given.

Equation (4.8) for $\mathrm{C} \rightarrow 0$ can be integrated once; the integration constant is zero due to the symmetry conditions. Our numerical integrations of the resulting equation show that the solutions are indistinguishable for cases when the slip coefficient $\beta=10^{-6}$ and $\beta=0$. This is consistent with Greenspan's (1978) observation (for the case $G=0, m=1, M=0$ ) that the imposition of slip is not necessary if one examines only the leading term of the small-C approximation.

If $\beta$ is set to zero, then the once-integrated evolution equation becomes

$$
\left(h_{x x}-G h\right)_{x}+\frac{3}{2} M \frac{h x}{h}=0 .
$$

Similarly,

$$
\left[\frac{1}{r}\left(r h_{r}\right)_{r}-G h\right]_{r}+\frac{3}{2} M \frac{h_{r}}{h}=0 .
$$





\section{Results: isothermal spreading}

When the system is isothermal, $M=0$, equation ( 5.1 ) can be solved, subject to the conditions of symmetry (3.16), contact (3.15) and constant volume (3.18). The result is

$$
h(x, t)=\frac{1}{2} \sqrt{G} \frac{\cosh x / G-\cosh a / G}{\sinh a \sqrt{ } G-a \sqrt{ } \cosh a \sqrt{ } G} .
$$

Similarly,

$$
h(r, t)=\frac{d G}{a} \frac{I_{0}(r / G)-I_{0}(a / G)}{I_{1}(a / G)-\frac{1}{2} a \sqrt{ } G I_{0}(a / G)},
$$

where $I_{n}$ is the modified Bessel function of the first kind.

Forms (6.1) are now substituted into the contact-angle conditions ( 3.17 ) and (3.19) to yield

$$
\begin{aligned}
\frac{1}{a_{t}^{m}}+\theta_{A} & =-h_{x}(a, t) \\
& =\frac{1}{2} G[a / G \operatorname{coth} a / G-1]^{-1}, \\
a_{t}^{\frac{1}{m}}+\theta_{A} & =-h_{r}(a, t) \\
& =\frac{G}{a} \frac{I_{1}(a / G) / I_{0}(a / G)}{\frac{1}{2} a \sqrt{ } G-I_{I}(a / G) / I_{0}(a / G)} .
\end{aligned}
$$

Equations (6.2) are differential equations for $a=a(t)$ subject to the initial condition $\mathrm{a}(0)=1$.

Case 1: $\Theta_{\mathrm{A}}>0, \mathrm{G}=0$.

The drop spreads to an equilibrium configuration. This is governed by equation $(6.2 p)$ with $a_{t}=0, a=a_{\infty}$, and $h_{x}$ replaced by its $G=0$ limit. The result is 


$$
\begin{aligned}
& a_{\infty}=\left(\frac{3}{2 \theta_{A}}\right)^{\frac{1}{2}}, \\
& a_{\infty}=\left(\frac{8}{\theta_{A}}\right)^{\frac{1}{3}} .
\end{aligned}
$$

Clearly, given the volume of the drop, the smaller the contact angle, the larger the final size. This final state is independent of exponent $m$. One can easily show also by perturbing equation (6.2) about the final state (6.3) that there is exponential approach to equilibrium. This is always the case for limited spreading, the spreading to $a=a_{\infty}<\infty$.

Case 2: $\theta_{\mathrm{A}}>0, \mathrm{G}>0$.

Gravity acting vertically downward will flatten the drop center and hence increase $a_{\infty}$. In fact, for small $G$,

$$
a_{\infty}-\left(\frac{3}{2 \theta_{A}}\right)^{\frac{1}{2}}\left(1+\frac{1}{30} G\right)
$$

and

$$
a_{\infty}-\left(\frac{8}{\theta_{A}}\right)^{\frac{1}{3}}\left(1+\frac{1}{72} G\right) .
$$

Figure 3 shows the evolution of the drop shapes with $\theta_{A}=0.25$ and $G=0$ and $G$ $=0.5$. Gravity both accelerates the spreading and increases $a_{\infty}$.

Figure 4 shows the instantaneous streamlines in the approach to steady state. The density of the streamlines indicates the speed. Notice that the fluid is flowing downward, from the summit towards the contact line, and particularly downward along the liquid/gas interface. 
Case 3: $\theta_{\mathrm{A}}=0, \mathrm{G}=0$.

Since $\theta_{\mathrm{A}}=0$, the drop will experience unlimited spreading, i.e., a $\rightarrow \infty$ as $t \rightarrow \infty$. When $G=0$, equation $(6.2 p)$ has the form

$$
a^{2} a_{t}^{\frac{1}{m}}=\frac{3}{2} \text {. }
$$

Hence, as $t \rightarrow \infty$,

$$
a-c_{m} t^{\frac{1}{2 m+1}}
$$

where

$$
c_{m}=\left[(2 m+1)\left(\frac{3}{2}\right)^{m}\right]^{\frac{1}{2 m+1}} .
$$

Similarly, in the axisymmetric case

$$
a^{3} a_{t}^{\frac{1}{m}}=8
$$

and for $t \rightarrow \infty$

$$
a-c_{m} t^{\frac{1}{3 m+1}}
$$

where

$$
c_{m}=\left[(3 m+1) 8^{m}\right]^{\frac{1}{3 m+1}} .
$$

For the exponent $m=1$ formulae $(6.6 p)$ and $(6.6 a)$ give $a \sim t^{1 / 3}$ and a $-t^{1 / 4}$, respectively. As shown in Table $I$, these behaviors, are those of Greenspan (1978). For the exponent $m=3$, these formulae give $a \sim t^{1 / 7}$ and $a \sim t^{1 / 10}$. The former agrees with the excision result of Tanner (1979) and agrees with his experiment. The latter agrees with the excision result of 
Tanner (1979) and Starov (1983) and the experiments of Tanner (1979), Cazabat and Cohen Stuart (1986) and Chen (1988).

The present results in both geometries support the use of the present uniform theory and the mobility exponent $\mathrm{m}=3$, for capillary-dominated spreading.

Case 4: $\theta_{\mathrm{A}}=0 . \mathrm{G}>0$.

Since $\theta_{A}=0$, the drop will experience unlimited spreading in that a $\rightarrow \infty$ as $t \rightarrow \infty$. If one inspects equation (6.2), one sees that gravity should have a profound effect on the spreading process. In case $3, h_{x}(a, t)$ was approximated for small $G$ with fixed a. However, when the spreading is unlimited, any fixed value of $G$, no matter how small, will eventually result in a $/ G$ becoming large; the limits $a+\infty, G \rightarrow 0$ and $G \rightarrow 0, a \rightarrow \infty$ are not equivalent.

When $a \sqrt{ } G$ is large, equation $(6.2 p)$ becomes

$$
a a_{t}^{\frac{1}{m}}=\frac{1}{2} \sqrt{G}
$$

so that as $t \rightarrow \infty$,

$$
a-d_{m} t^{\frac{1}{m+1}}
$$

where

$$
d_{m}=\left[\left(\frac{1}{2} \sqrt{G}\right)^{m}(m+1)\right]^{\frac{1}{m+1}}
$$

Similarly, in the axisymmetric case, equation (6.2a) gives

$$
a^{2} a_{t}^{\frac{1}{m}}=2 \sqrt{G}
$$

so that as $t \rightarrow \infty$,

$$
a-d_{m} t^{\frac{1}{2 m+1}}
$$


where

$$
d_{m}=[2 \sqrt{G(2 m+1)}]^{\frac{1}{2 m+1}}
$$

For the exponent $m=1$ formulae $(6.9 p)$ and $(6.9 a)$ give $a-t^{1 / 2}$ and $a \sim t^{1 / 3}$, respectively. These results are new. For the exponent $m=3$ these formulae give $a-t^{1 / 4}$ and $a-t^{1 / 7}$, respectively. Both of these differ slightly from the excision results of Lopez et al. (1970). The axisymmetricspreading data of Cazabat and Cohen Stuart (1986) seem to agree better with the form $t^{1 / 8}$ than our $t^{1 / 7}$, suggesting that the mobility exponent $m=3.5$ might give a better fit for their data.

The present results in both geometries support the use of the present uniform theory with a mobility exponent m equal to or somewhat greater than 3 . The results show that the long-time spreading with gravity is substantially accelerated compared to the case $G=0$, given the same mobility exponent $\mathrm{m}$. The fact that gravity promotes spreading is no surprise. What seems paradoxical is the fact that a thin drop, which at early times is negligibly affected by gravity, will be greatly affected by gravity later when it is much thinner. Cazabat and Cohen Stuart (1986) have conducted spreading experiments on smooth surfaces with axisymmetric drops under isothermal conditions. They report during the spreading two different scaling laws depending on whether or not gravity is important. In a first phase of the spreading process they found capillary effects to be dominant while, in a second phase, for larger $t$, the influence of gravity seems to be controlling the process. This behavior can be understood as follows. At early times the hydrostatic pressures are small compared to capillary forces, but as the drop becomes flatter and flatter, the curvature goes to zero faster than the thickness and the small hydrostatic pressures ultimately dominate. 
Figure 5 illustrates this behavior; it shows our numerical solution of equation (6.2p) for $m=1$ and various $G$. Note that as $t \rightarrow \infty$, the drop width a $\sim t^{1 / 2}$ when $G \neq 0$; compare this to the $G=0$ result, $a-t^{1 / 3}$. Note as well that if one excludes initial transients, that the $G=0$ behavior $a-t^{1 / 3}$ is valid for early times even if $G \neq 0$. These results are consistent with the observations of Cazabat and Cohen Stuart (1986). They find that in practice the two ranges are not separated by values of the instantaneous Bond number $G^{(a)}$,

$$
G^{(a)}=\frac{\rho g a^{2}(t)}{\sigma}
$$

viz. $G^{(a)}<1$ meaning capillary domination and $G^{(a)}>1$ meaning gravity domination. They are demarked by fixed values of $a^{-3 / 2} a_{t}$. 


\section{Results: nonisothermal spreading}

When the plate is heated or cooled, there are thermocapillary forces that render the drop dynamic; fluid flow is always present. The shape of the drop is governed for $C \rightarrow 0$ by equation (5.1). When this is integrated once we find for the plane geometry that

$$
h_{x x}-G h+\frac{3}{2} M \ln h=-s_{1}
$$

where $s_{1}$ is a constant. Clearly, both $h$ and $h_{x}$ are regular at $x=a$ while the curvature undergoes rapid variations there due to strong thermocapillary gradients over very thin regions. The same singularity would be present if slip were retained; the factor $3 / 2$ would then be replaced by unity.

Similarly, in the axisymmetric case

$$
\frac{1}{r}\left(r h_{r}\right)_{r}-G h+\frac{3}{2} M \ln h=-s_{1} .
$$

Equation (7.1p) can be multiplied by $h_{x}$ and integrated again, to yield

$$
\frac{1}{2}\left(h_{x}^{2}-G h^{2}\right)+\frac{3}{2} M h(\ln h-1)=-s_{1} h+s_{2}
$$

where $s_{2}$ is another constant.

In all that follows, the Marangoni number $M$ is nonzero giving thermocapillary-driven motions in the drop. For reason of clarity we neglect gravitational effects $(G=0)$. For small values of $M$, perturbation theory can be used to solve equation (7.1), subject to the symmetry (3.16) and contact conditions ( 3.15 ), for constant volume (3.18). The asymptotic representation of $\mathrm{h}$ is 


$$
\begin{aligned}
& h(x, t)-\frac{3}{4}\left\{\frac{a^{2}-x^{2}}{a^{3}}+M\left[2\left(a^{2}+x^{2}\right) \ln (2 a)-\frac{4}{3}\left(a^{2}-x^{2}\right)\right.\right. \\
& \left.\left.-(a-x)^{2} \ln (a-x)-\left(a+x^{2}\right) \ln (a+x)\right]\right\} \\
& h(r, t)-\frac{2}{\pi} \frac{a^{2}-r^{2}}{a^{4}}+\frac{3}{8} M\left[\sum_{n=1}^{\infty}\left(\frac{a}{n}\right)^{2}\left\{1-\left(\frac{r}{a}\right]^{2 n}\right\}\right. \\
& \left.+\left(a^{2}-r^{2}\right)\left\{\ln \left(\frac{a^{2}-r^{2}}{a^{2}}\right)-\frac{3}{2}\right\}\right] .
\end{aligned}
$$

We substitute forms (7.2) into the contact-angle conditions ( 3.17 ) and (3.19) to obtain

$$
\begin{aligned}
a_{t}^{1 / m+\theta_{A}} & =-h_{x}(a, t), \\
& -\frac{3}{2 a^{2}}\left(1-\frac{a^{3}}{3} M\right), \\
a_{t}^{1 / m}+\theta_{A} & =-h_{r}(a, t), \\
& -\frac{4}{\pi a^{3}}\left(1-\frac{3 \pi a^{4}}{32} M\right) .
\end{aligned}
$$

Equations (7.3) are approximations of differential equations for $a=a(t)$ subject to the initial conditions $a(0)=1$.

Case 1: ${ }_{\mathrm{A}}>0, \mathrm{G}=0$

The drop spreads to an equilibrium configuration with $a=a_{\infty}$ and the fluid flow approaches a steady state. From equations (7.3) it follows for small M that

$$
a_{\infty}-\left(\frac{3}{2 \theta_{A}}\right)^{\frac{1}{2}}\left(1-\frac{1}{4} M\right)
$$

and 


$$
a_{\infty} \sim\left(\frac{8}{\theta_{A}}\right)^{\frac{1}{2}}\left(1-\frac{1}{8} M\right)
$$

By comparison with equations $(6.4)$ it is seen that thermocapillarity on a heated plate acts oppositely to gravity.

The drop on a heated plate $(M>0)$ exhibits a circulation driven by thermocapillarity as shown in Figure 6. The higher surface tension at the drop summit and a lower surface tension at the edges are responsible for the liquid/gas interface being "pulled" towards the drop summit. The flow is inward toward the center where it turns around. This turning is driven by a pressure gradient in which there is a higher pressure at the center and this deforms the drop; the interface steepens near the center and flattens near the edge. This effect is similar to the deformation of the surface in a slot caused by a recirculating thermocapillary flow as explained by Sen and Davis (1982). By conservation of mass, the edge is at a position $a_{\infty}<a_{\infty 0}=\left(\frac{3}{2 \theta_{A}}\right)^{1 / 2}$ as shown in equation $(7.4 p)$.

A comparison of the transient behaviors for $M=0$ and $M=0.2$ is shown in Figure 7. The heating decelerates the spreading and limits the final drop size. The evolution to the final shape involves the spreading flow down the interface as shown in Figure 4 and a counterflow up the interface driven by thermocapillarity. The result is the complex flow field shown in Figure 8 . Equations (7.2), (7.3) and (7.4) also hold for the case of a cooled plate, $M<0$. In such a situation the direction of the thermocapillary flow is reversed, resulting in a flatter drop with extended edges $a_{\infty}>a_{\infty 0}$ as given in equation (7.4). Figure 9 shows the drop evolution for two values of $M$. For large enough $M$, the drop dips into the center giving a non-convex drop which no longer approaches a steady shape; it spreads to infinity. This is 
caused by the thermocapillary-driven flow pulling liquid outward along the 1iquid/gas interface as shown in Figure 10. Here the thermally-driven flow aids the pure-spreading flow, shown in Figure 4.

Case 2: $\quad{ }_{\mathrm{A}}=0, \mathrm{G}=0$

In the absence of thermocapillarity, the drop with advancing contact angle zero will always spread to infinity, i.e. $a \rightarrow \infty$ as $t \rightarrow \infty$.

For small $M$ and $\theta_{A}=0$ equations (7.3) have to be integrated to give an approximate representation of $a(t)$. For $m=1$ we integrate equations (7.3p) numerically with $a(0)=1$; the solutions are shown in Figure 11 . When $M=0$, there is the usual a $\sim t^{1 / 3}$ of isothermal spreading. Weak heating $(M>0)$ retards the spreading and, as we shall see, causes the drop with $\Theta_{A}=0$ to cease spreading with $a_{\infty}<\infty$. Weak cooling $(M<0)$ promotes the spreading.

Case 3: Critical spreading, $G=0$

Given that cooling can promote spreading, one can ask whether a cooled drop with $\theta_{A}>0$ can be made to spread to infinity. Figure 12 shows our numerical solutions to equation (7.3p) for $\theta_{A}=0.25$ and when $M<-0.1$ there seems to be spreading to infinity. We therefore investigate the critical combinations of $M$ and $\theta_{A}$ that result in unlimited $\left(a_{\infty}=\infty\right)$ or limited spreading $\left(a_{\infty}<\infty\right)$.

In equations (7.3), we set $a=a_{\infty}, a_{t}=0$ and obtain an algebraic system for $a_{\infty}$,

$$
\begin{aligned}
& M a_{\infty}^{3}+2 \Theta_{A} a_{\infty}^{2}-3=0 \\
& M a_{\infty}^{4}+\frac{8}{3} \Theta_{A} a_{\infty}^{3}-\frac{32}{3 \pi}=0
\end{aligned}
$$


A numerical scheme, based on Laguerre's method, is employed to solve for the zeros of this polynomial with real coefficients as the parameters are varied in the ranges $-1 \leq M \leq 1$ and $0 \leq{ }_{A} \leq 1$. Figure 13 shows the positive real solutions for $a_{\infty}=f(M)$ for two different values of the advancing contact angle $\Theta_{\mathrm{A}}$. From these plots we see that any increase of the $M$ decreases the final drop width $a_{\infty}$. Given a fixed drop volume, an increase in $\theta_{A}$ will result in a smaller final drop width. This feature may be seen in Figure 13 by comparing the curves obtained for $\theta_{A}=0$ and $\theta_{A}=0.5$. Figure 13a further shows that the simultaneous 1 imit $M \rightarrow 0$ and $\theta_{A} \rightarrow 0$ involves a singular behavior as $a_{\infty} \rightarrow \infty$. In particular we can characterize the singularity by applying separate limits, viz.

$$
\begin{aligned}
M \ll \theta_{A} \ll 1: & a_{\infty} \propto \theta_{A}^{-1 / 2}, \\
& a_{\infty} \propto \Theta_{A}^{-1 / 3}, \\
\Theta_{A} \ll M \ll 1: & a_{\infty} \propto M^{-1 / 3}, \\
& a_{\infty} \propto M^{-1 / 4} .
\end{aligned}
$$

From Figure $13 \mathrm{~b}$ we can infer that, although the singularity is preserved for $M=0$, we have a real solution for $a_{\infty}$ existing within a range of negative M. A left limit point terminates this range. This limit point is associated with a change in the number of real solutions of equation (7.5). (We presume that the lower branch existing to the right side of the limit point is stable, while the upper one is unstable.) In the range to the left of the limit point no real positive solution of equation (7.5) exists and as a consequence we expect unlimited spreading. We find from equation (7.5) that there is unlimited spreading if 


$$
\begin{aligned}
& M<M_{L P}=-\frac{4 / 2}{9} \theta_{A}^{3 / 2}, \\
& M<M_{L P}=-\left\{\left(\frac{\pi}{2}\right)^{1 / 3} \theta_{A}^{4 / 3} .\right.
\end{aligned}
$$




\section{Discussion and conclusion}

We have considered the spreading of Newtonian-viscous liquid drops on a heated or cooled horizontal plate. We used lubrication theory to reduce the governing equations to a set of evolution equations for the interface shape $h$ and the contact-line position a as defined in Figure 1. This system includes the effects of viscosity, surface tension, gravity, thermocapillarity and wetting characteristics and generalizes Greenspan (1978) to non-isothermal systems with gravity and with power-law forms for $\theta=F(U)$. Both twodimensional and axisymmetric drops have been examined for small capillary numbers.

\section{Isothermal spreading:}

When gravity acts downward toward the plate, static drops $\left(\Theta_{A}>0\right)$ are flattened at their centers and hence extend outward further than they would in a gravity-free environment.

When the drop spreads finitely far, the final approach is exponential. However, at earlier times or if the drop spreads to infinity $\left(\Theta_{A}=0\right)$, the drop spreading follows a power law. We have found that gravitational forces can be very important on the scaling law at long times when the drop is very flat, even though they are negligible at earlier times when the drop is thicker. This prediction is in accord with the observations of Cazabat and Cohen Stuart (1987). Table I, shows if one takes the mobility exponent $\mathrm{m}=3$ so that $\left(\theta-\theta_{\mathrm{A}}\right) \propto \mathrm{U}^{1 / 3}$, that there is excellent agreement between the present theory and the existing isothermal experiments, a result that gives validation to both the $\mathrm{m}=3$ model and the uniform theory used. This uniform theory supposes that all local (molecular) physics at the contact line is incorporated into the function $F$ of $\theta=F(U)$. 


\section{Nonisothermal spreading:}

The influence of the heat transport on a spreading drop is substantial. The thermocapillary forces have their strongest effect near the contact line where they compete with the effects of wetting and capillarity. They retard (augment) the spreading when the plate is heated (cooled). They further create strong deformations in the interface shape near the contact line; the curvature is logarithmically infinite there in the model posed.

The general conclusion is that heating (cooling) the plate retards (augments) spreading. When $\theta_{A}=0$, the isothermal drop will spread to infinity. If the plate is heated, and $\Theta_{A}=0$ still, then the drop will spread only finitely far. If $\Theta_{A}>0$, the isothermal drop will spread finitely far. If the plate is cooled sufficiently, and $\theta_{\mathrm{A}}>0$ still, then the drop will spread to infinity. We examined the final states and determined the precise conditions of heating/cooling versus $\theta_{A}$ for which unlimited or finite spreading occurs. These results show the existence of a mechanism for the thermal control of spreading, one that is quite sensitive to the thermal gradients. This is a potentially important practical tool.

There are a number of generalizations of the present work that would be interesting. A direct numerical simulation of the evolution equation could determine effects present when $\mathrm{C}$ is not small. More general thermal boundary conditions might be appropriate especially if one wishes to model more precisely the contact-line region. The heat transport in our model in conduction dominated. Thus, the thermocapillary circulation transports little heat, though, this transport might become substantial if the heating were more intense. Convective effects might be incorporated into the evolution equation 
as well as variations with temperature of the viscosity. We have a uniformlyheated plate so that the contact angle $\theta_{\mathrm{A}}$, a local value, is constant. If the plate had non-uniform temperature, then one might have to account for $\Theta_{A}=\theta_{A}(T)$, or more generally, $\theta=F(U, T)$.

The present work suggests experiments of several varieties. One class could involve qualitative questions such as augmentation or retardation of spreading by heat transfer. A second class could involve quantitative explorations into power laws for spreading, and interface shapes.

\section{Acknowledgements}

P.E. is pleased to acknowledge the support of the Kernforschungszentrum Karlsruhe in his visit to Northwestern University. S.H.D. gratefully acknowledges the support of the U.S. Department of Energy, Division of Basic Energy Sciences, through Grant No. DEFG02-86ER13641. 


\section{References}

Cazabat, A. M. and Cohen Stuart, M. A. 1986 Dynamics of wetting: effects of surface roughness, J. Phys. Chem. 90, 5845-5849.

Chen, J.-D. 1988 Experiments on a spreading drop and its contact angle on a solid, J. Coll. Interf. Sci. 122, 60-72.

Dussan V., E. B. and Davis, S. H. 1974 On the motion of a fluid-fluid interface along a solid surface, J. Fluid Mech. 65, 71-95.

Dussan V., E. B. 1976 The moving contact line: the slip boundary condition, J. Fluid Mech. 77, 665-684.

Dussan V., E. B. 1979 On the spreading of liquids on solid surfaces: static and dynamic contact lines, Ann. Rev. Fluid Mech. 11, 371-400.

de Gennes, P. G. 1985 Wetting: statics and dynamics, Rev. Mod. Phys. 57, 827-863.

Greenspan, H. P. 1978 on the motion of a small viscous droplet that wets a surface, J. Fluid Mech. 84, 125-143.

Hocking, L. M. 1981 Sliding and spreading of thin two-dimensional drops, Q. J. Mech. App1. Math. 34, 37-55.

Hocking, L. M. 1983 The spreading of thin drops by gravity and capillarity, Q. J. Mech. App1. Math. 36, 55-69.

Lopez, J., Miller, C. A. and Ruckenstein, E. 1976 Spreading kinetics of liquid drops on solids, J. Coll. Interf. Sci. 56, 460-468.

Rosenblat, S. and Davis, S. H. 1985 How do liquid drops spread on solids?, Frontiers in Fluid Mech. (ed. S. H. Davis and J. L. Lumley), Springer, Heidelberg, 171-183.

Schwartz, A. M. and Tejeda, S. B. 1972 Studies of dynamic contact angles on solids, J. Coll. Interf. Sci. 38, 359-375.

Sen, A. K. and Davis, S. H. 1982 Steady thermocapillary flows in two dimensional slots, J. Fluid Mech. 121, 163-184.

Tanner, L. H. 1979 The spreading of silicone oil on horizontal surfaces, J. Phys. D: Appl. Phys. 12, 1473-1484. 
TABLE I

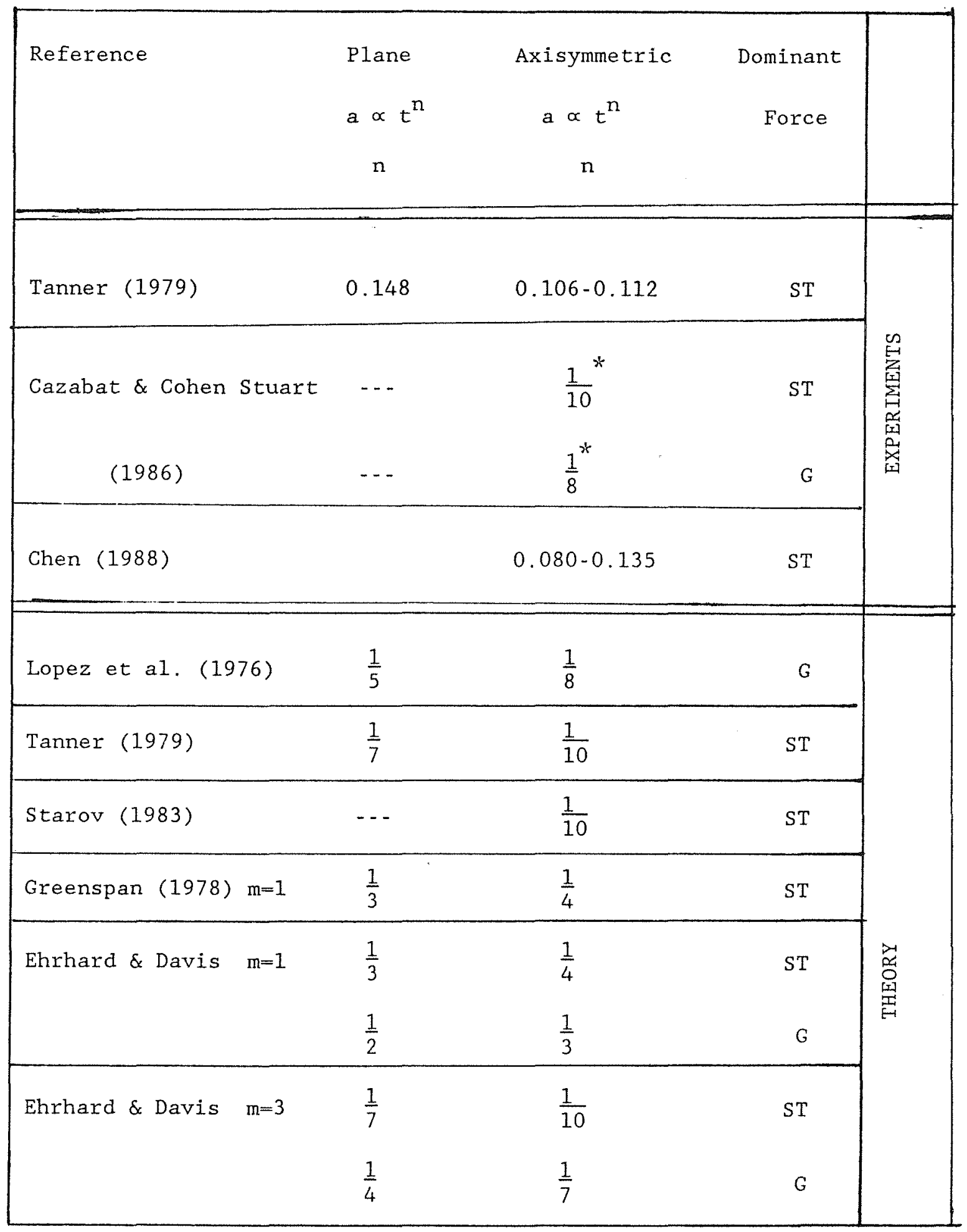


Figure Captions

Figure 1: $\quad$ Sketch of the problem geometry.

Figure 2: Typical measurements of contact angle after Dussan V. (1979); Symbols represent experimental data, solid lines correspond to various mobility exponents $m$ in model equation (2.19).

Figure 3: Isothermal spreading $(M=0)$; evolution of the drop shapes with $(G=0.5)$ and without $(G=0)$ gravity for $\theta_{A}=0.25$.

Figure 4: Isothermal spreading $(M=0)$ : evolution of the stream function with $\theta_{A}=0.25$, and $G=0.05$. Given are instantaneous streamlines in steps $\Delta \psi=0.01$.

Figure 5: Isothermal spreading $(M-0)$ : positions of the contact lines as function of time for various $G$ with $\Theta_{A}=0.25$.

Figure 6: Nonisothermal spreading: steady drop shape and streamlines $M=0.2, G=0$. The horizontal and vertical axes are rescaled using units appropriate to the steady drop under isothermal conditions.

Figure 7: Nonisothermal spreading: evolution of the drop shapes for isothermal $(M=0)$ conditions and for a heated plate $(M=0.2)$ with $\theta_{\mathrm{A}}=0.25$ and $\mathrm{G}=0$.

Figure 8: Nonisothermal spreading: evolution of the stream function with $M=0.2, \theta_{A}=0.25$ and $G=0$. Given are instantaneous streamlines in steps $\Delta \psi=0.01$. 
Figure 9: Nonisothermal spreading: evolution of the drop shapes for a slightly $(M=-0.05)$ and a more intensely $(M=-0.10)$ cooled plate with $\Theta_{\mathrm{A}}=0.25$ and $G=0$.

Figure 10. Nonisothermal spreading: evolution of the stream function with $\mathrm{M}=-0.1, \Theta_{\mathrm{A}}=0.25$ and $\mathrm{G}=0$. Given are instantaneous streamlines in steps $\Delta \psi=0.01$.

Figure 11. Nonisothermal spreading: effect of the Marangoni number on spreading with $\Theta_{A}=0, G=0, m=1$ and various $M$.

Figure 12: Critical spreading: positions of the contact lines as functions of time with $\theta_{A}=0.25, G=0$ and various $M$.

Figure 13: Critical spreading: final drop widths $a_{\infty}$ as functions of Marangoni number $M$ for $G=0$ and for two different advancing contact angles (a) $\Theta_{\mathrm{A}}=0$, and (b) $\Theta_{\mathrm{A}}=0.5$.

Table caption

Table I: Isothermal spreading results. The symbols ST and G denote surface-tension and gravity dominance, respectively. The asterisk indicates that error bars were not given. 


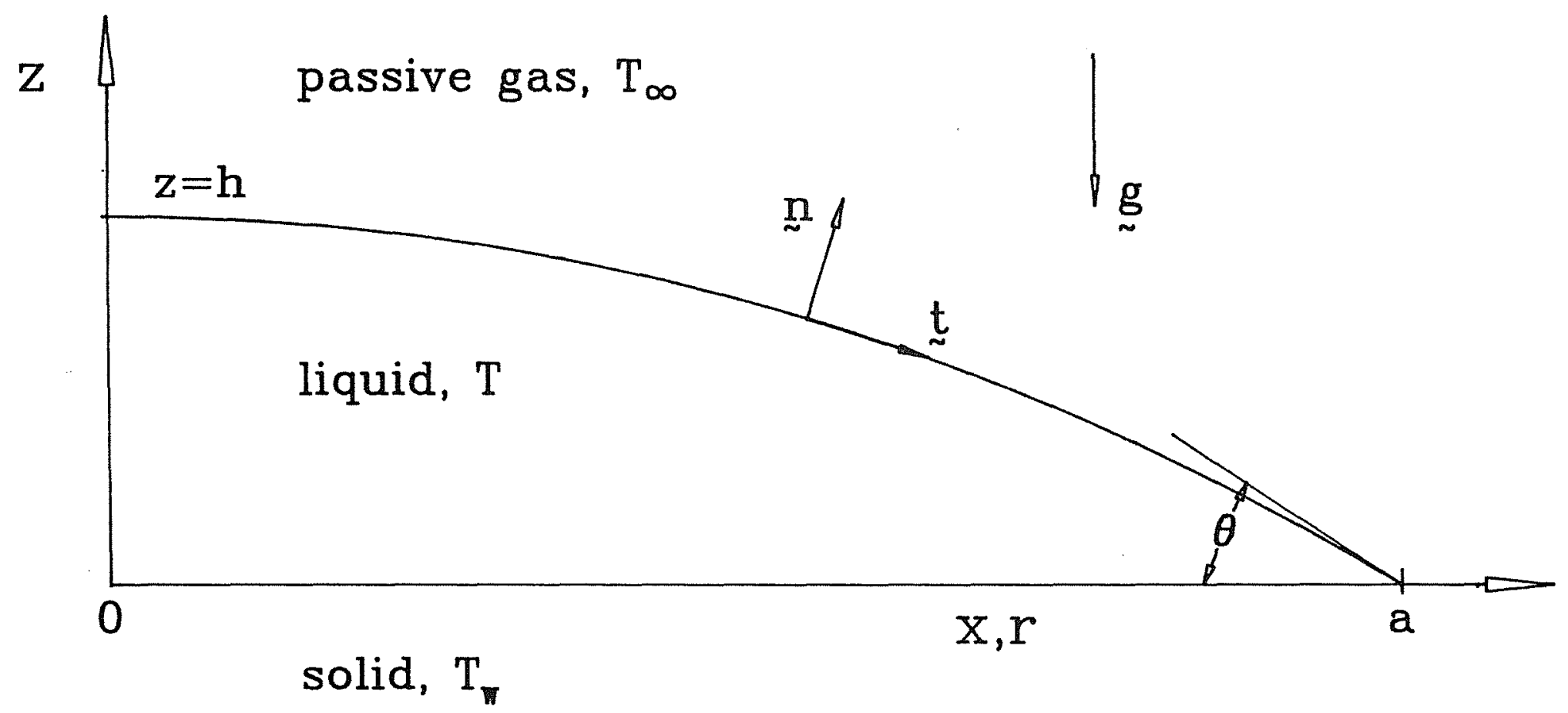

Figure 1: 


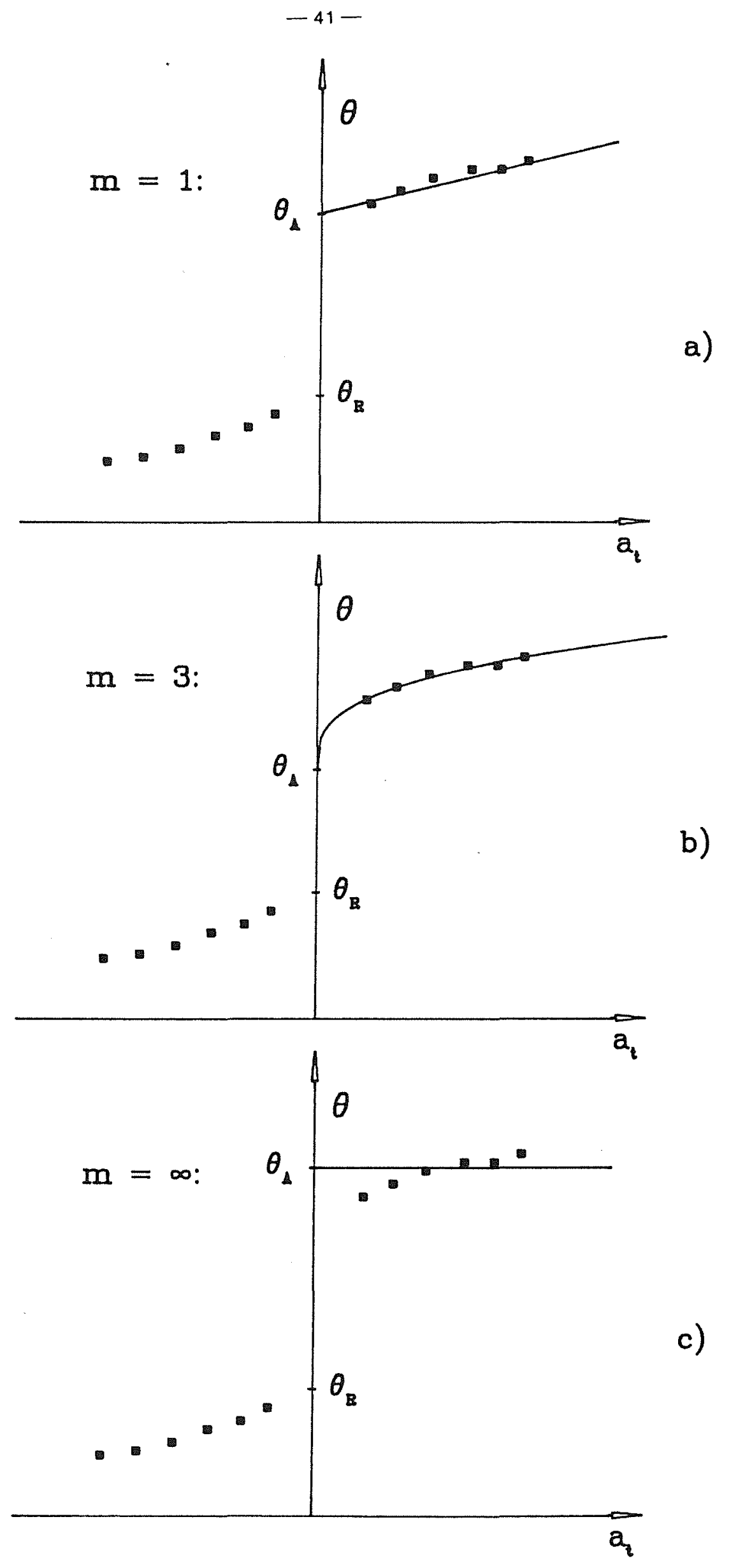

Figure 2: 


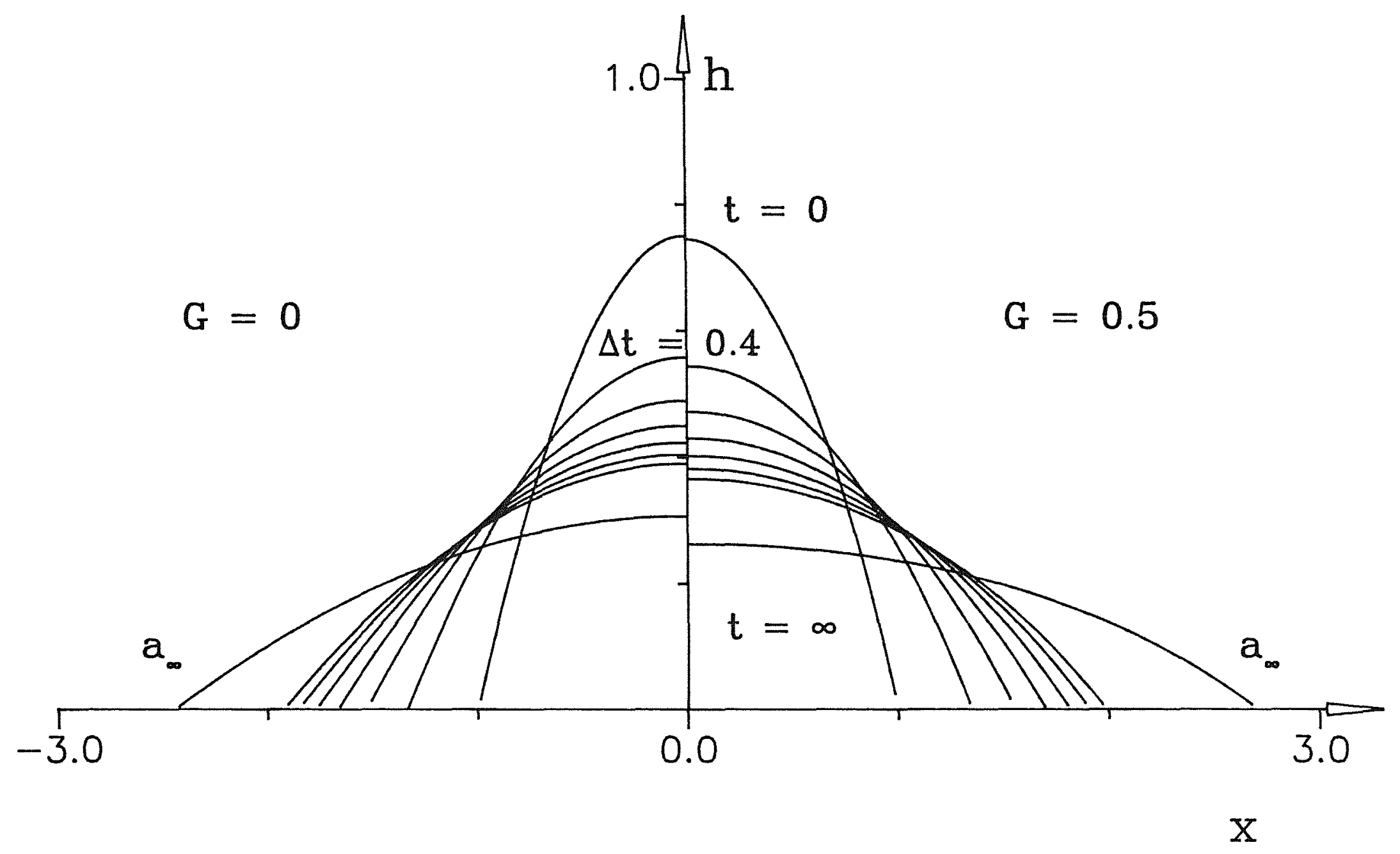

Figure 3: 


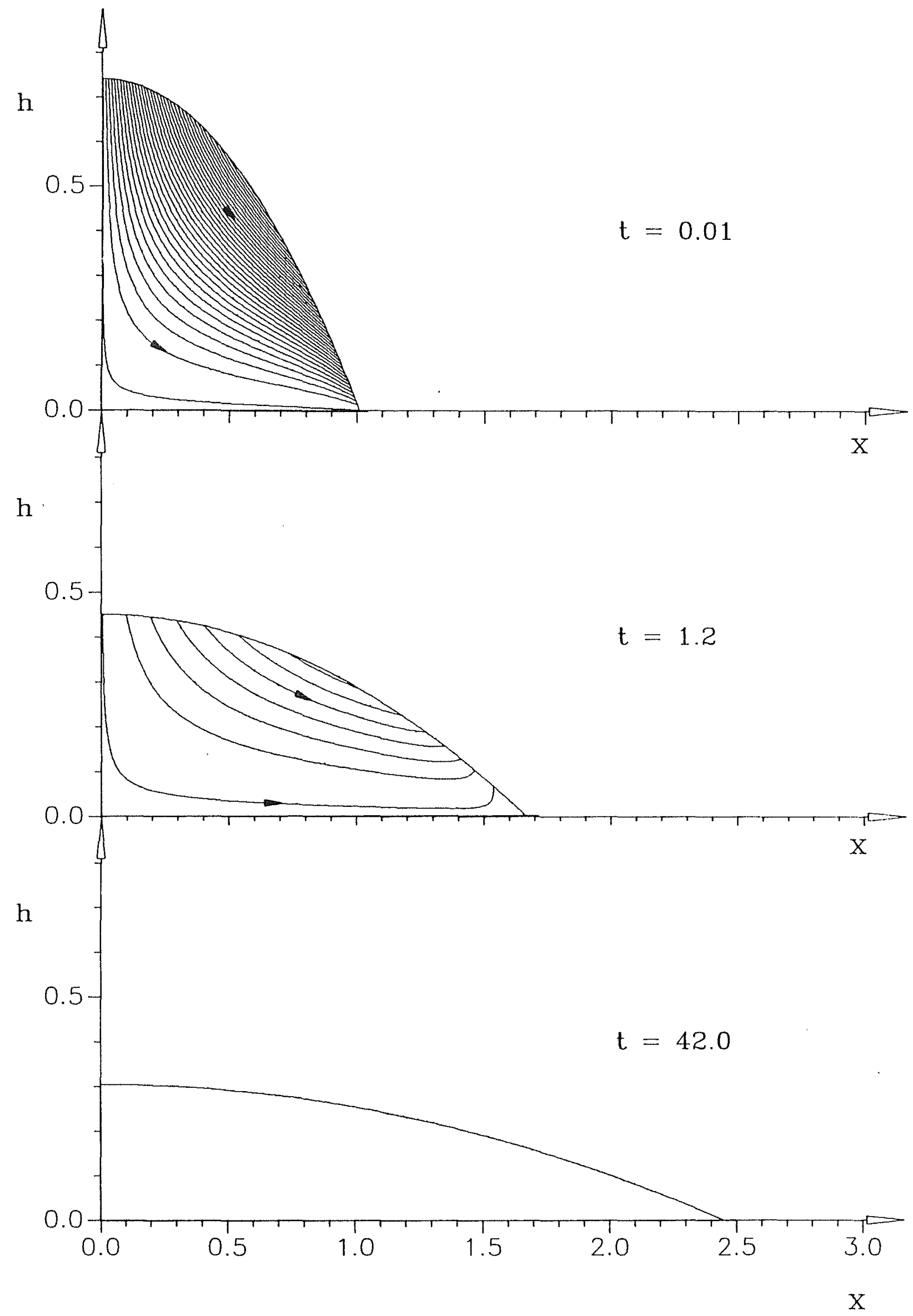

Figure 4: 


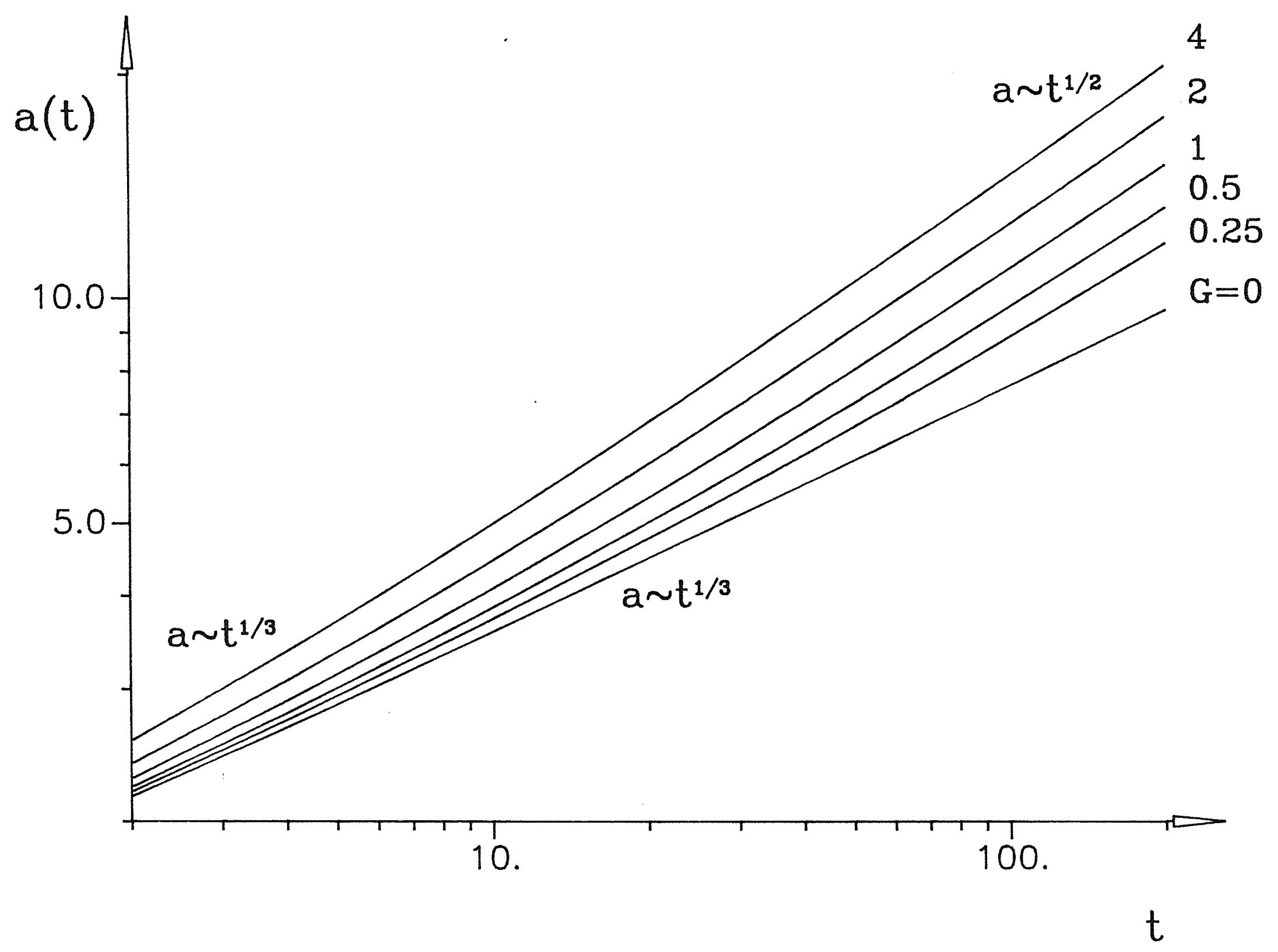

Figure 5: 


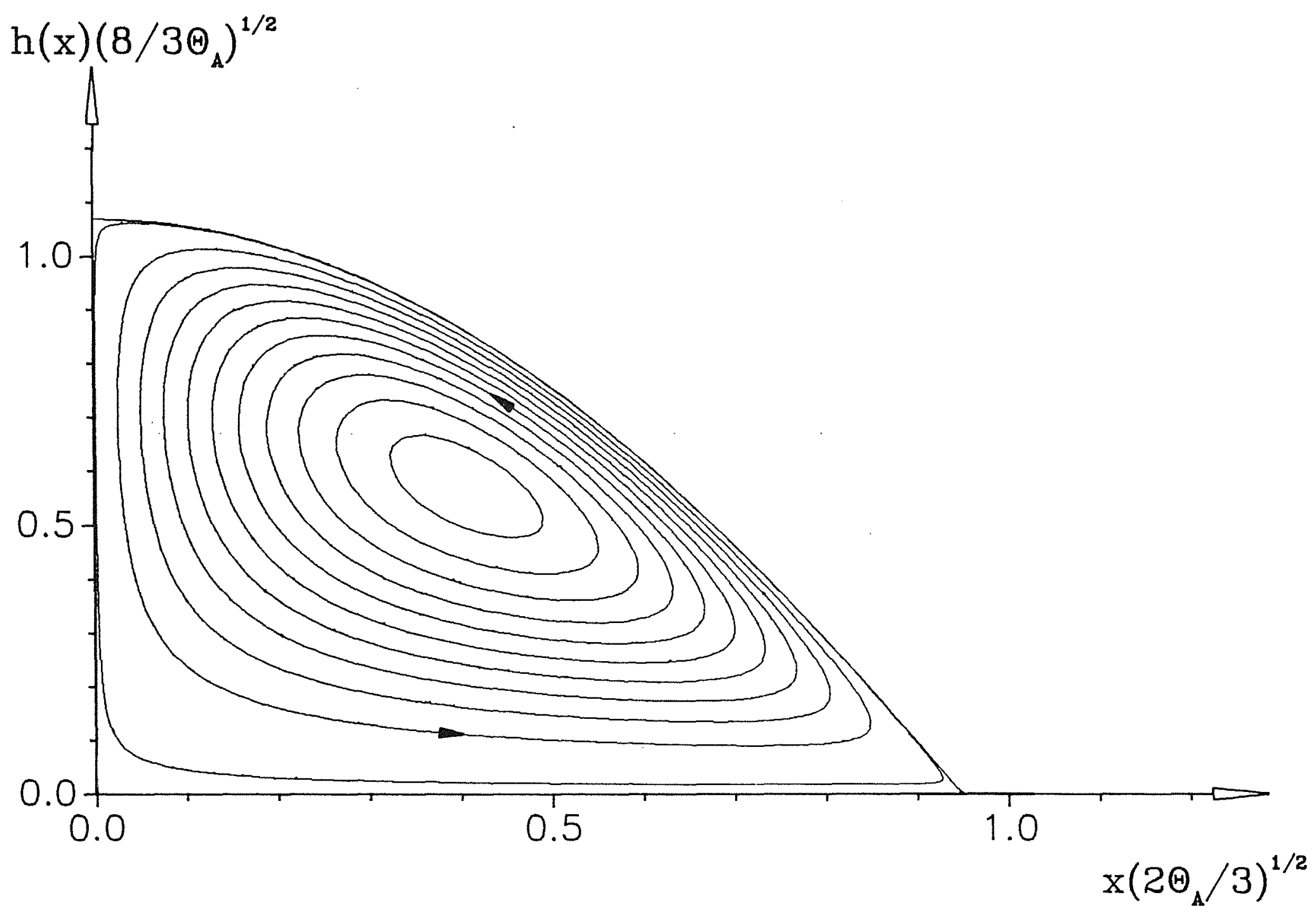

Figure 6: 


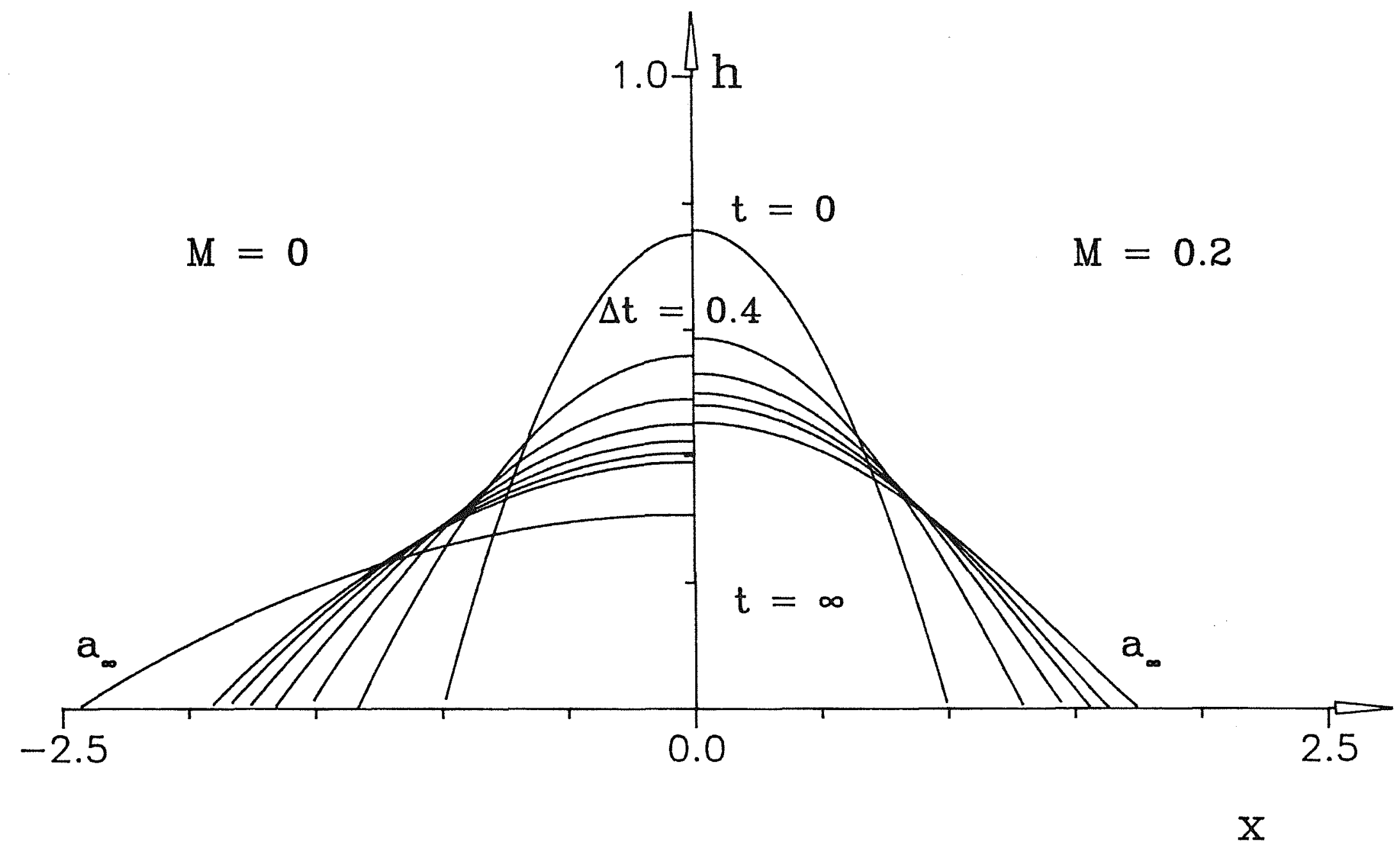

Figure 7: 


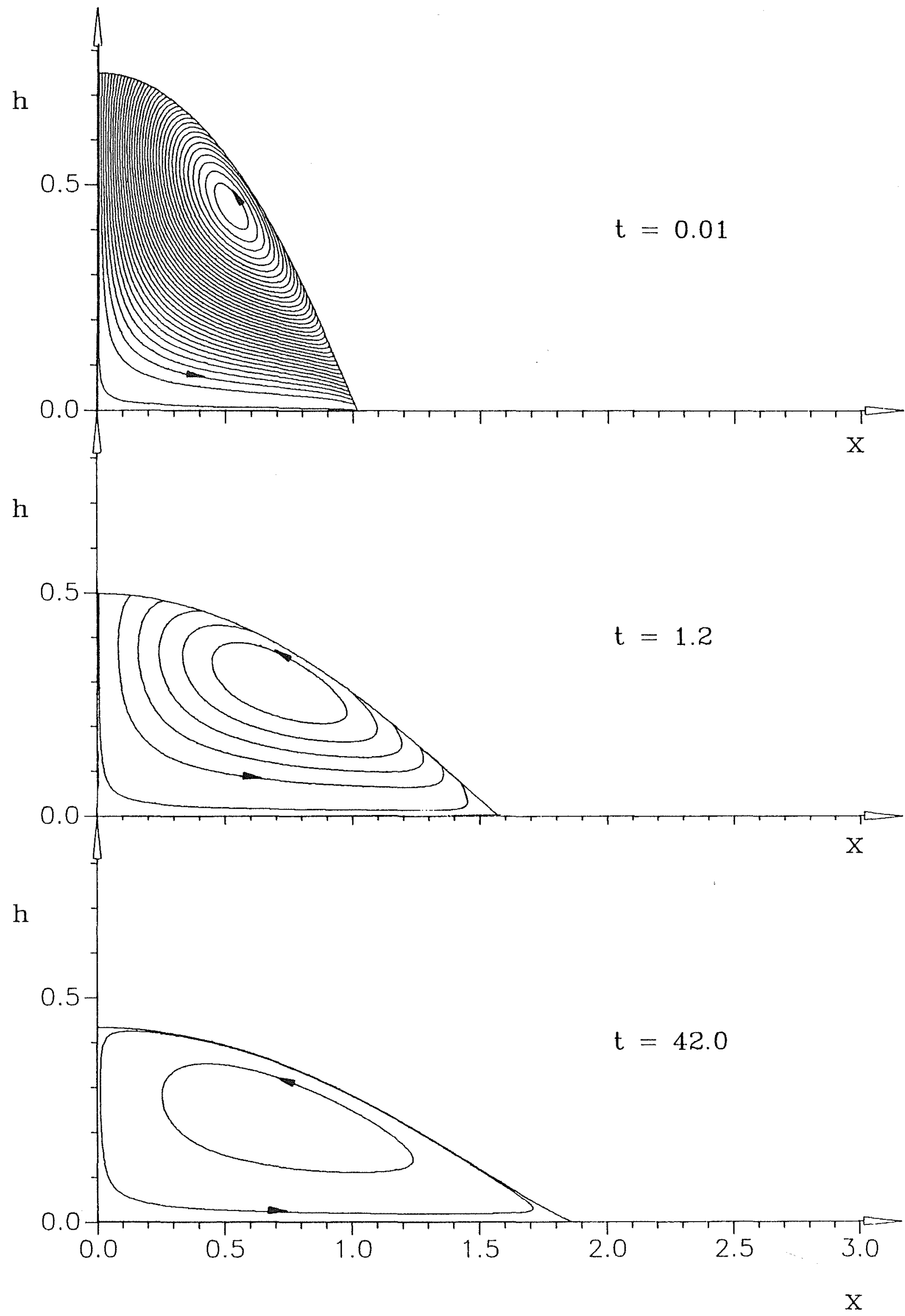

Figure 8: 


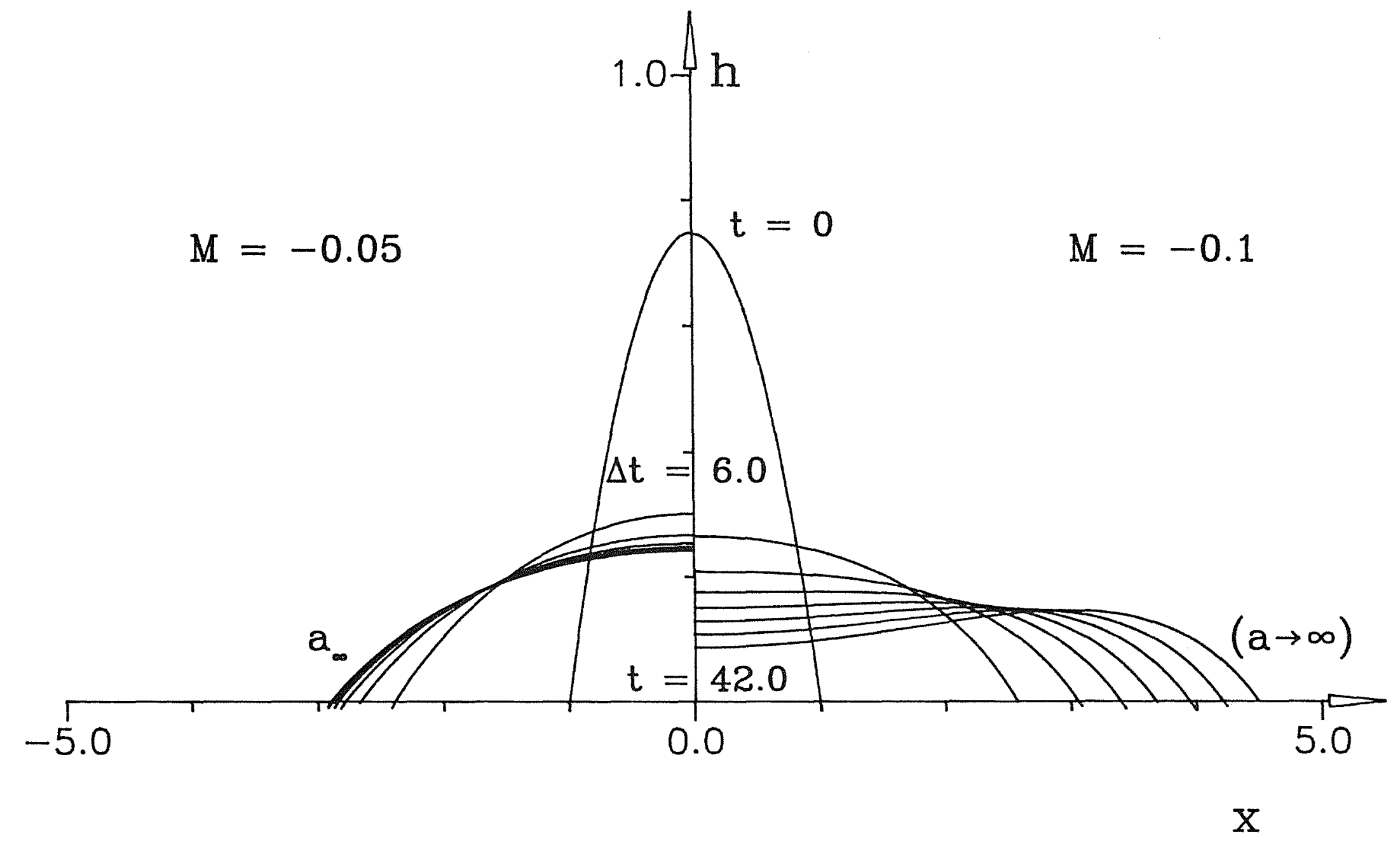

Figure 9: 


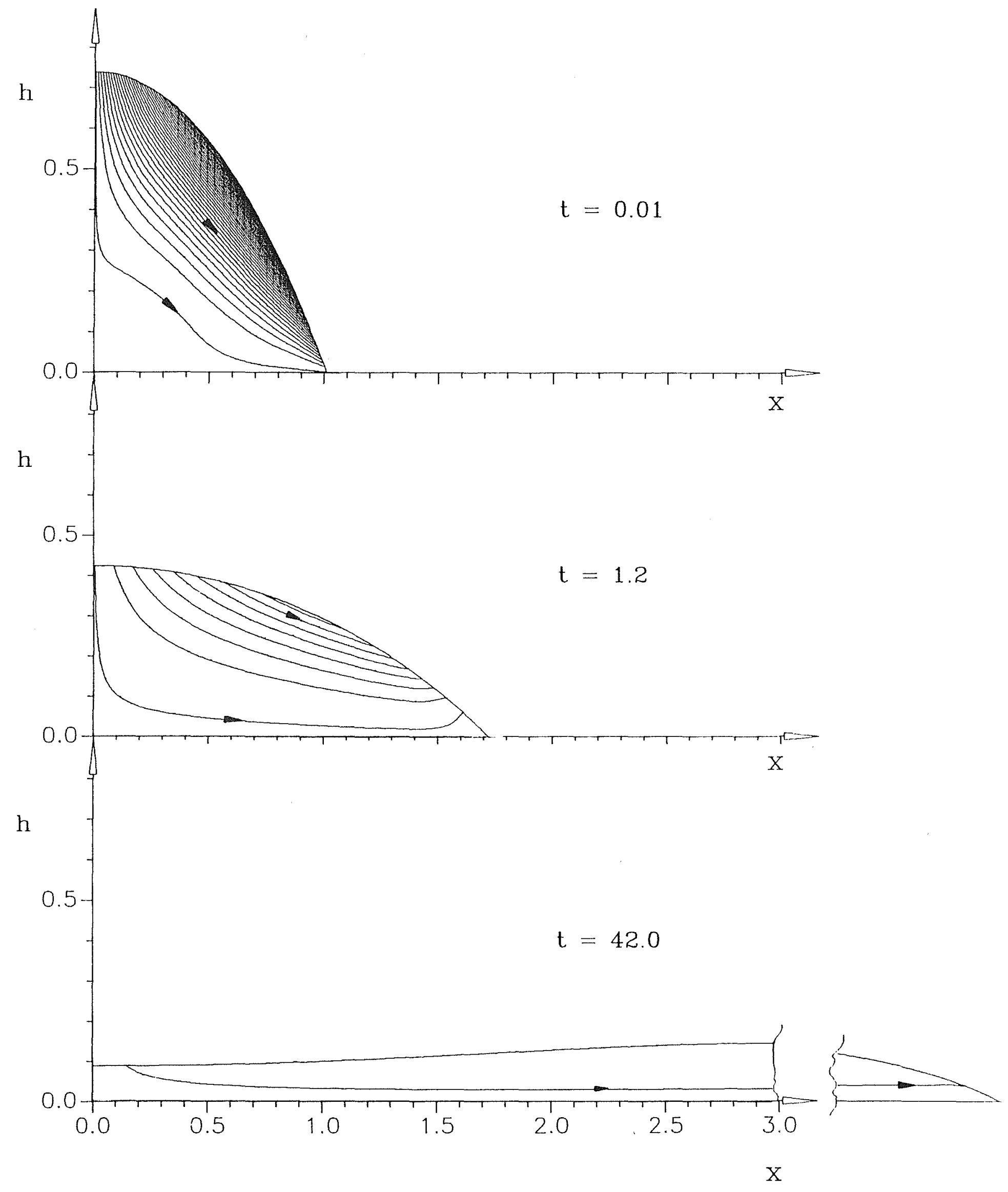

Figure 10: 


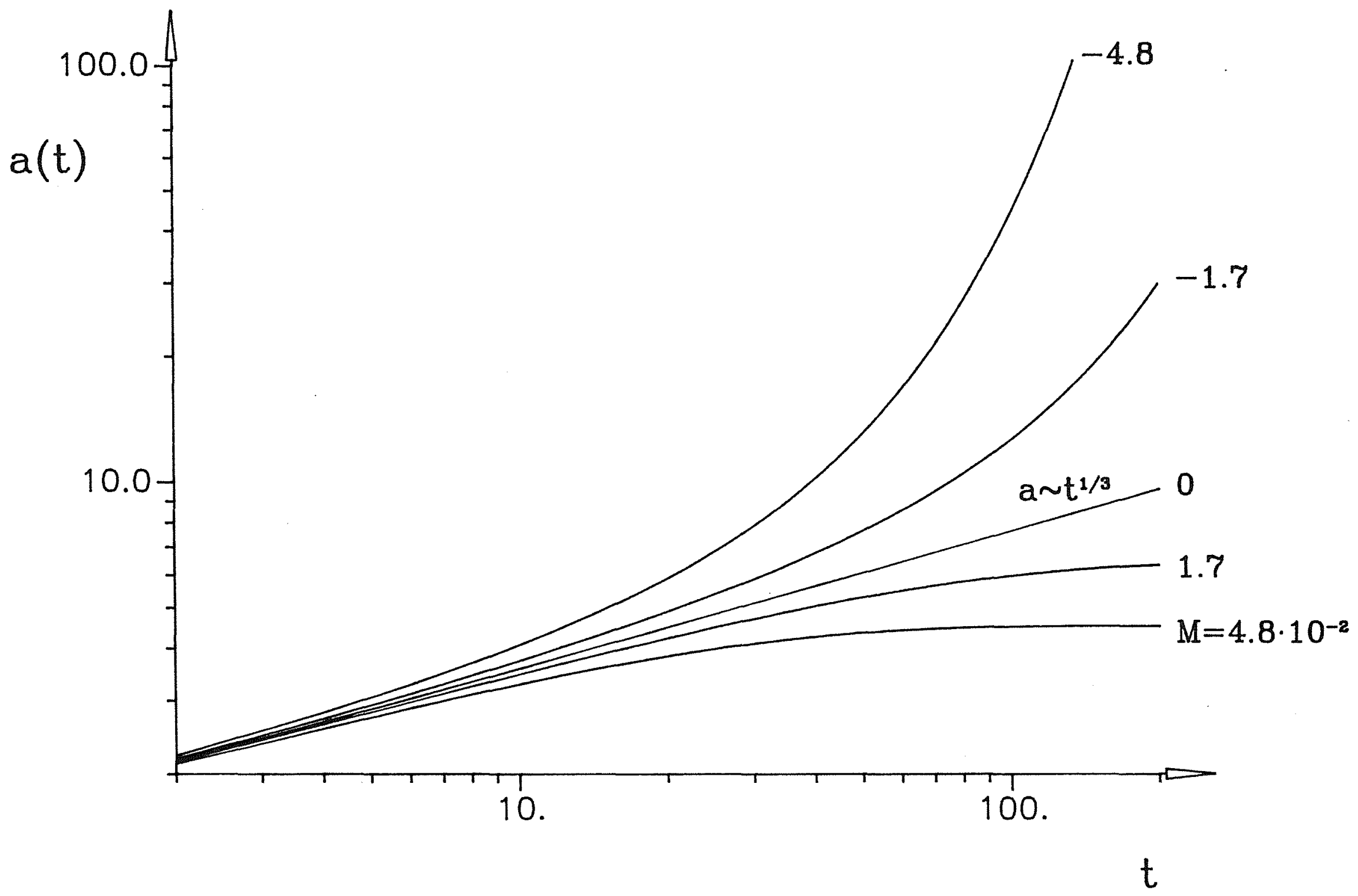

Figure 11: 


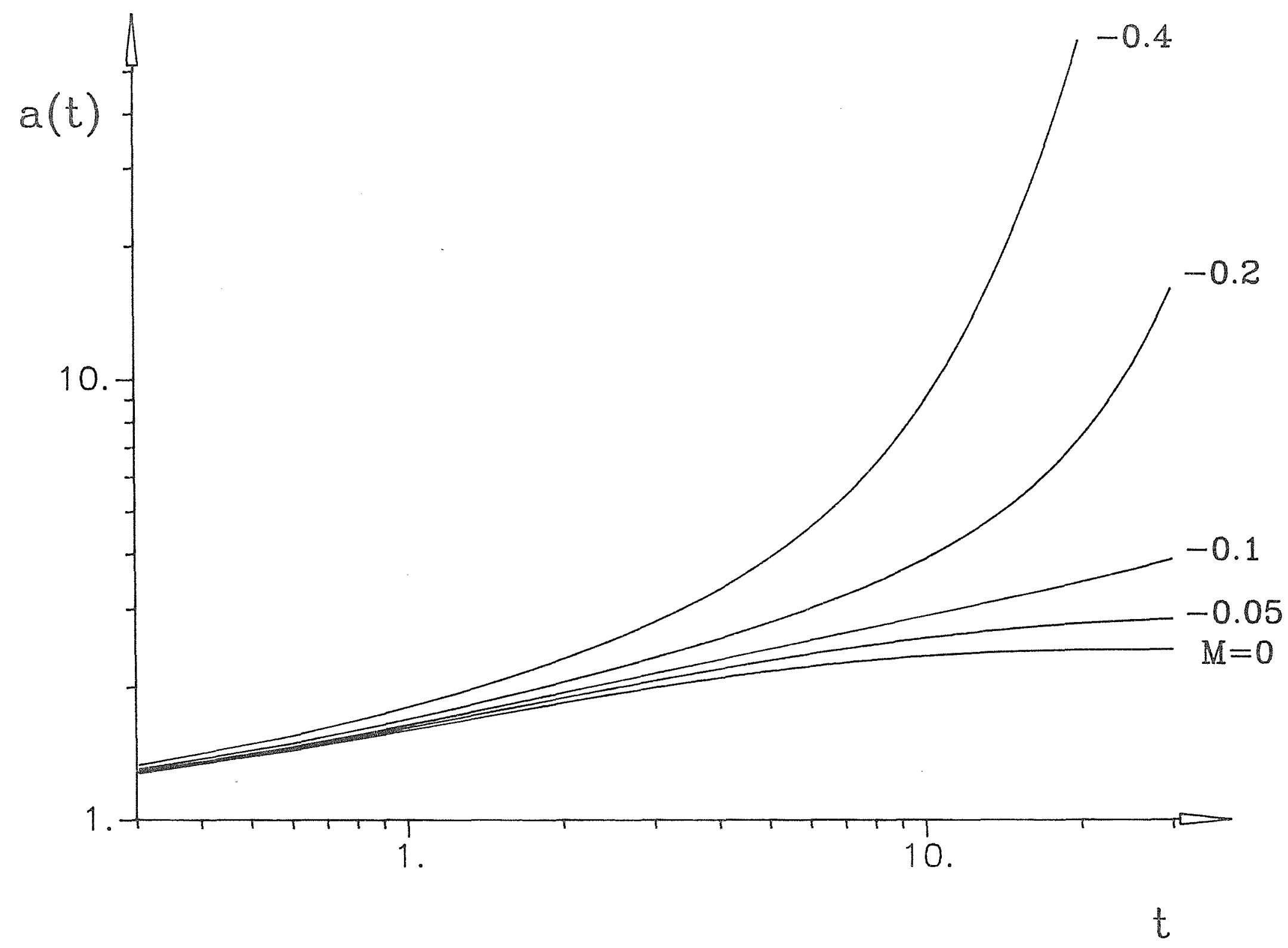

Figure 12: 


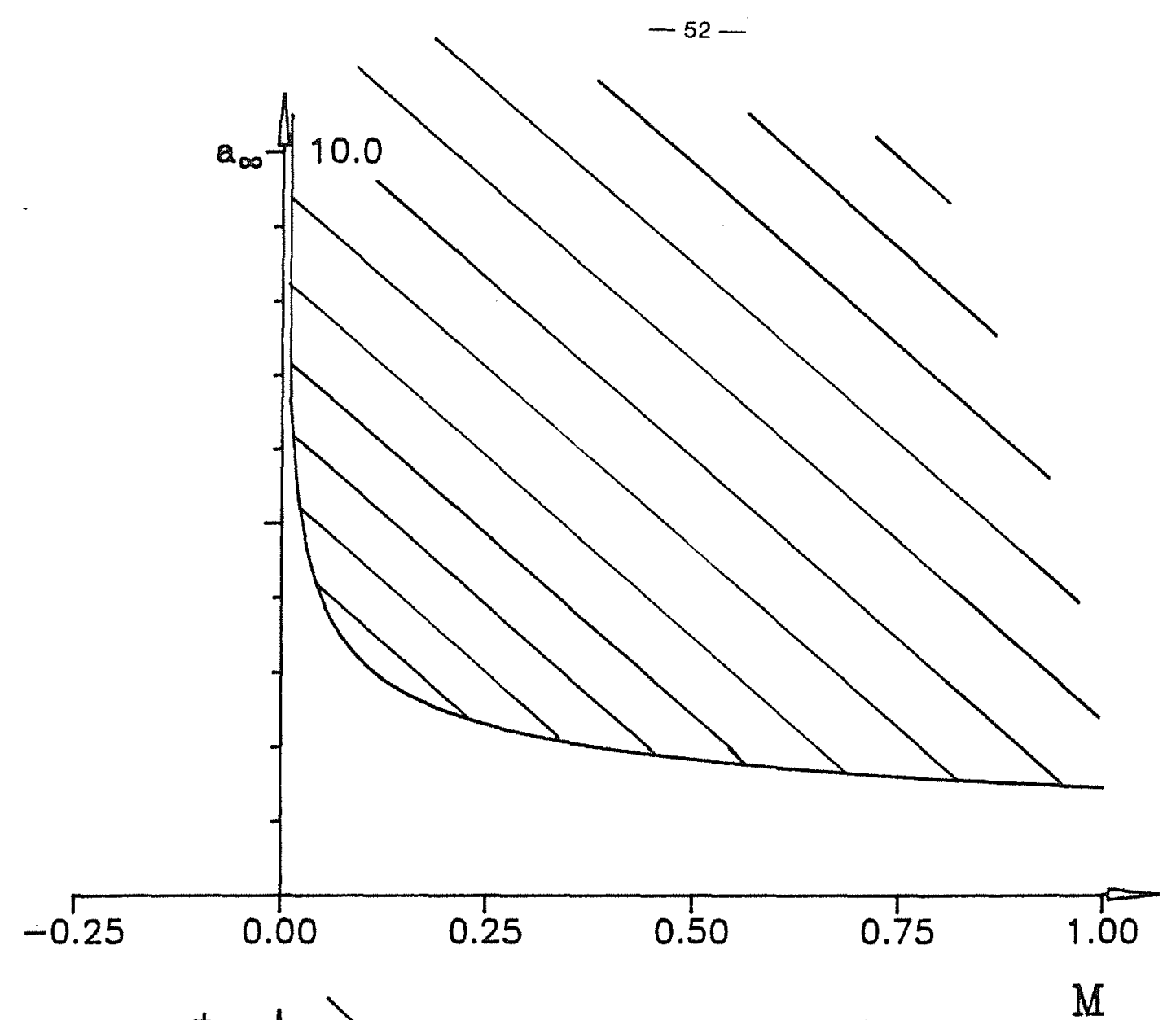

a)

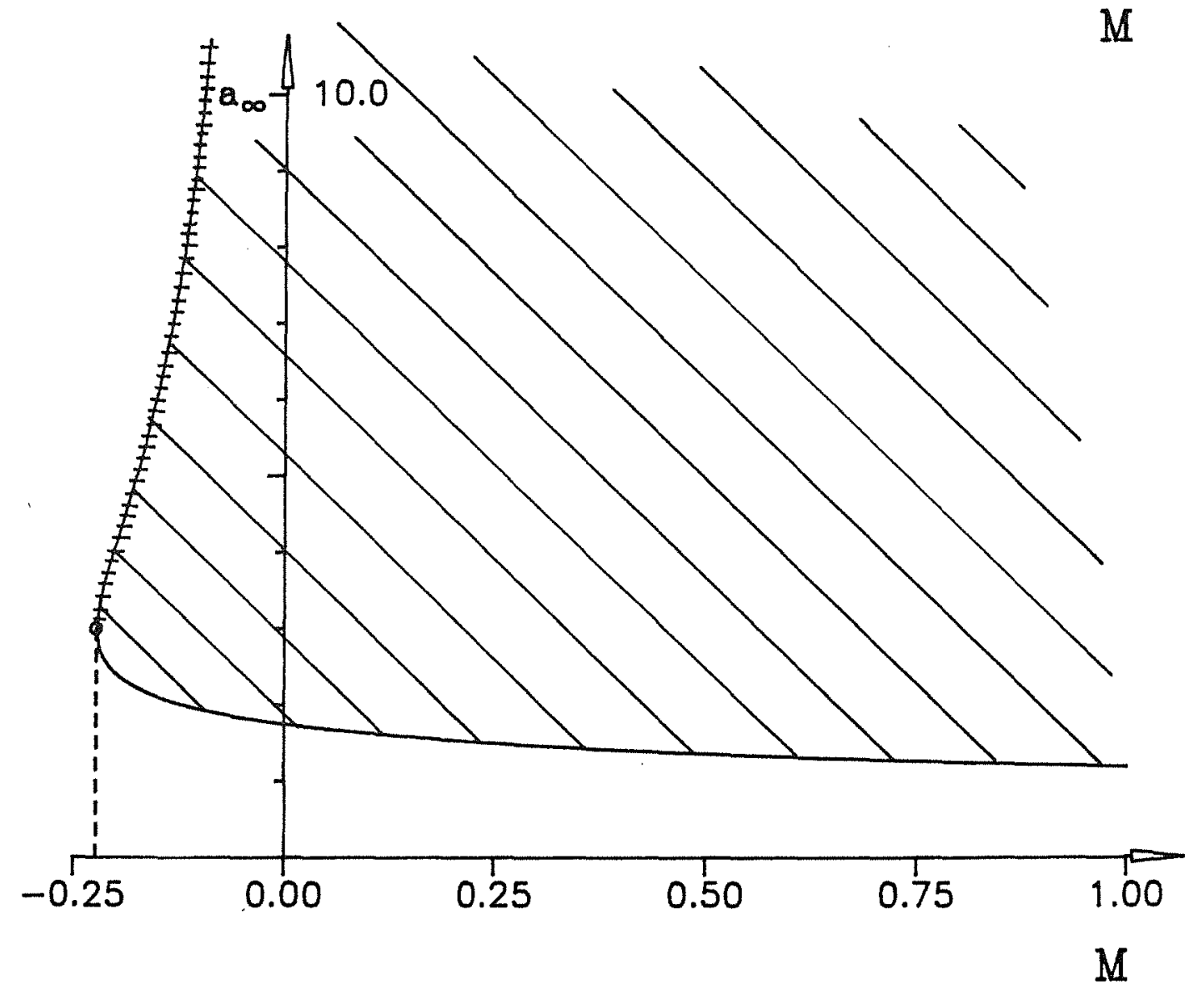

b)

Figure 13: 